University of New Hampshire

University of New Hampshire Scholars' Repository

$11-1-2013$

\title{
The Magnetic Electron Ion Spectrometer (MagEIS) Instruments Aboard the Radiation Belt Storm Probes (RBSP) Spacecraft
}

\author{
J. B. Blake \\ P. A. Carranza \\ S. G. Claudepierre \\ J. H. Clemmons \\ W. R. Crain
}

See next page for additional authors

Follow this and additional works at: https://scholars.unh.edu/physics_facpub

Part of the Physics Commons

\section{Recommended Citation}

Blake, J. B.; Carranza, P. A.; Claudepierre, S. G.; Clemmons, J. H.; Crain, W. R.; Dotan, Y.; Fennell, J. F.; Fuentes, F. H.; Galvan, R. M.; George, J. S.; Henderson, M. G.; and Lalic, M., "The Magnetic Electron Ion Spectrometer (MagEIS) Instruments Aboard the Radiation Belt Storm Probes (RBSP) Spacecraft" (2013). Space Science Reviews. 153.

https://scholars.unh.edu/physics_facpub/153

This Article is brought to you for free and open access by the Physics at University of New Hampshire Scholars' Repository. It has been accepted for inclusion in Physics Scholarship by an authorized administrator of University of New Hampshire Scholars' Repository. For more information, please contact Scholarly.Communication@unh.edu. 


\section{Authors}

J. B. Blake, P. A. Carranza, S. G. Claudepierre, J. H. Clemmons, W. R. Crain, Y. Dotan, J. F. Fennell, F. H. Fuentes, R. M. Galvan, J. S. George, M. G. Henderson, and M. Lalic 


\title{
The Magnetic Electron Ion Spectrometer (MagEIS) Instruments Aboard the Radiation Belt Storm Probes (RBSP) Spacecraft
}

\author{
J.B. Blake · P.A. Carranza · S.G. Claudepierre - J.H. Clemmons · W.R. Crain Jr. • \\ Y. Dotan · J.F. Fennell • F.H. Fuentes · R.M. Galvan · J.S. George • M.G. Henderson • \\ M. Lalic · A.Y. Lin • M.D. Looper · D.J. Mabry • J.E. Mazur • B. McCarthy • \\ C.Q. Nguyen • T.P. O'Brien • M.A. Perez • M.T. Redding • J.L. Roeder • \\ D.J. Salvaggio • G.A. Sorensen · H.E. Spence · S. Yi • M.P. Zakrzewski
}

Received: 15 October 2012 / Accepted: 9 May 2013 / Published online: 7 June 2013 (C) The Author(s) 2013. This article is published with open access at Springerlink.com

\begin{abstract}
This paper describes the Magnetic Electron Ion Spectrometer (MagEIS) instruments aboard the RBSP spacecraft from an instrumentation and engineering point of view. There are four magnetic spectrometers aboard each of the two spacecraft, one low-energy unit (20-240 keV), two medium-energy units (80-1200 keV), and a high-energy unit (800$4800 \mathrm{keV})$. The high unit also contains a proton telescope (55 keV-20 MeV).

The magnetic spectrometers focus electrons within a selected energy pass band upon a focal plane of several silicon detectors where pulse-height analysis is used to determine if the energy of the incident electron is appropriate for the electron momentum selected by the magnet. Thus each event is a two-parameter analysis, an approach leading to a greatly reduced background.

The physics of these instruments are described in detail followed by the engineering implementation. The data outputs are described, and examples of the calibration results and early flight data presented.
\end{abstract}

Keywords Relativistic electron sensors - Energetic magnetospheric particles · Acceleration · Transport and loss of radiation belt particles

J.B. Blake $(\bowtie)$ · P.A. Carranza · S.G. Claudepierre · J.H. Clemmons · W.R. Crain Jr. · Y. Dotan · J.F. Fennell · F.H. Fuentes · R.M. Galvan · J.S. George · M. Lalic · A.Y. Lin · M.D. Looper ·

D.J. Mabry · J.E. Mazur · C.Q. Nguyen · T.P. O'Brien · M.A. Perez · M.T. Redding · J.L. Roeder ·

D.J. Salvaggio · G.A. Sorensen · S. Yi · M.P. Zakrzewski

Space Science Applications Laboratory, The Aerospace Corporation, El Segundo, CA 90245, USA

e-mail: jbernard.blake@aero.org

M.G. Henderson

Los Alamos National Laboratory, PO Box 1663, Los Alamos, NM 87545, USA

B. McCarthy

Environment Test and Assessment Department, The Aerospace Corporation, El Segundo, CA 90245, USA

H.E. Spence

Center for Earth, Oceans, and Space, University of New Hampshire, Durham, NH 03824, USA 
Fig. 1 The four magnetic spectrometers are shown in this cutaway drawing as mounted on the aft deck of a RBSP spacecraft. Each unit is connected to a thermal control surface on the down Sun side of the spacecraft by a thermal strap, shown in purple in the drawing

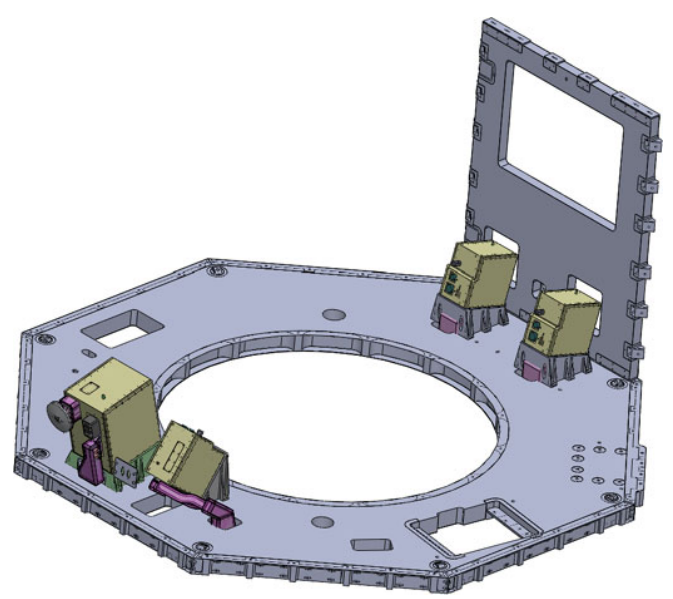

\section{Introduction}

An overarching objective of the RBSP mission is to obtain a deep understanding of the physics of the acceleration of electrons to relativistic energies in the radiation belts of the Earth. To this end a suite of four magnetic electron spectrometers aboard each of the two RBSP spacecraft will measure the differential fluxes, energies, and angular distributions of electrons from $20 \mathrm{keV}$ to $5 \mathrm{MeV}$. They have been designed to do so with only a small, determinable and correctible background during an extreme event such as occurred during March 1991, and to do so with a significant performance margin.

Magnetic electron spectrometers have been fielded by Aerospace aboard several satellite missions since the early 1960s when they were used to measure the intense fission electron fluxes injected into the magnetosphere by the high-altitude nuclear tests of the United States and the Soviet Union. The instrumentation described here is a substantial evolution of the earlier spaceflight investigations using current, vastly improved magnetic materials, silicon detectors and electronic components.

\section{Overview}

There are four MagEIS spectrometers on each of the two spacecraft. The spectrometers are mounted on the aft deck (anti-solar) of the satellite as shown in the cutaway drawing (Fig. 1). A low-energy, a medium energy, and a high-energy magnetic spectrometer view out a side of the spacecraft at 75 degrees to the satellite spin axis, and a second medium unit views out the bottom of the spacecraft at 35 degrees to the spin axis.

The MagEIS suite also contains a silicon-detector telescope that measures the differential fluxes, energies, and angular distributions protons from $60 \mathrm{keV}$ to $20 \mathrm{MeV}$, and helium and oxygen ions from a few hundred $\mathrm{keV} / \mathrm{nuc}$ to a few $\mathrm{MeV} /$ nuc. This telescope is contained in the high-energy magnetic spectrometer enclosure. 
Fig. 2 The basic magnetic spectrometer configuration is shown schematically

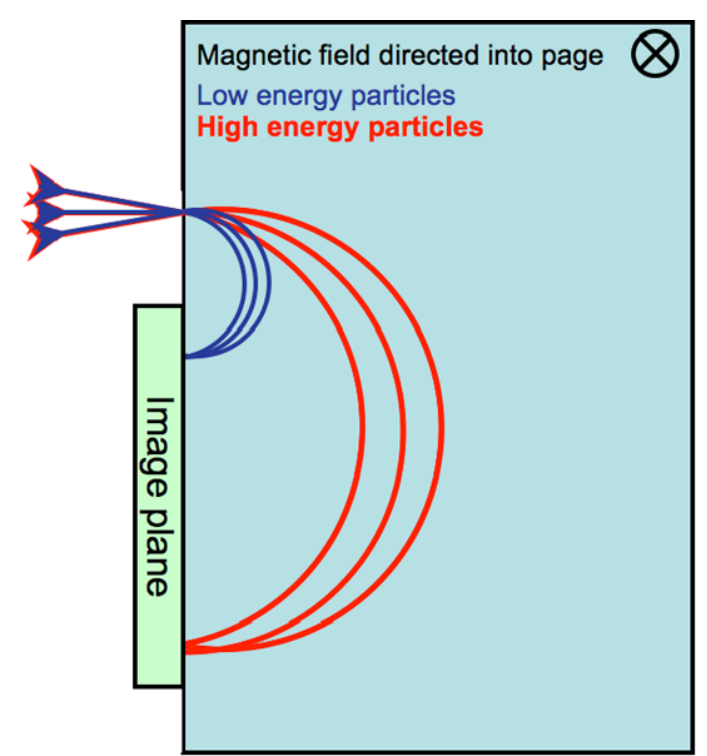

\section{Instrument Design Details-Magnetic Electron Spectrometers}

\subsection{Overview}

Electrons enter through a collimator defining the field of view of $10^{\circ} \times 20^{\circ}$ as shown in Fig. 2. The magnetic field inside the chamber is uniform, and focuses electrons on a linear strip of nine silicon detectors after the velocity vector of incoming electrons has been rotated through $180^{\circ}$. First order focusing occurs in the plane of the spectrometer; there is no focusing in the vertical plane. Focusing in the transverse plane occurs because the chord length subtending angles near $180^{\circ}$ in a circle does not change rapidly with change in angle subtended - the chord length is similar to the diameter. Anti-scatter baffling is placed at the top and bottom of the magnetic chamber.

The charge and mass of an electron are unique, and therefore no other magnetospheric particles are focused upon the detector plane. This feature is of paramount importance for instrument performance. No magnetospheric ions can enter through the aperture and strike the electron detectors. Furthermore each detector only sees those electrons in its pass band, unlike the case for a silicon-detector telescope where all electrons pass through the front detector(s) of the telescope. This situation has many critical benefits including removal of unwanted particles, major reduction of system dead time, and a substantial diminution in the radiation damage to the silicon detectors. The distance along the detector plane from the entrance aperture where a given electron is focused is a function of the electron momentum. The energy deposited in the silicon detector upon which the electron is focused determines the incident electron energy. Thus a two-parameter analysis is made for every electron. Only those electrons whose deposited energy is appropriate for the momentum determined by the position along the focal plane are valid events.

\subsection{Silicon Detector Configurations}

The detector thickness for each of the three-spectrometer types was selected based upon the maximum electron energy measured by a given spectrometer. Micron Semiconductor Ltd., 
Fig. 3 The Low and Medium Spectrometers have a nine-pixel focal plane

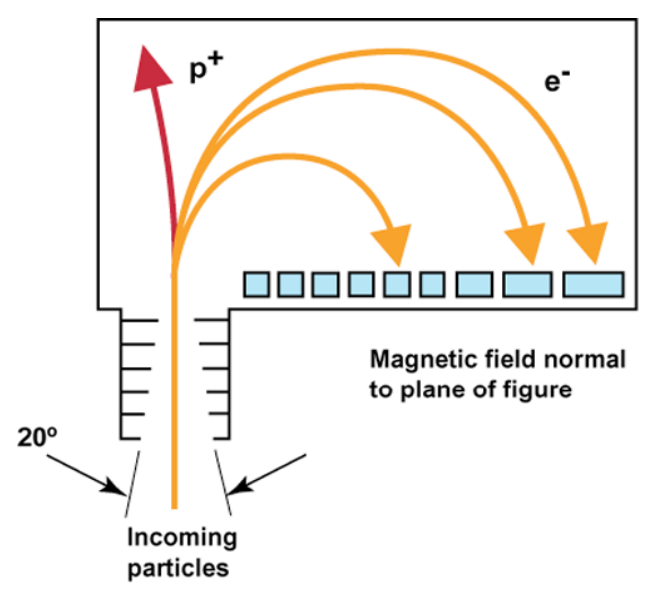

Lancing, England, fabricated all of the electron focal-plane detectors. Detectors consist of one $0.5 \mathrm{~mm}$, and two stacked $1.5 \mathrm{~mm}$ thick pieces of silicon for the Low and Medium detectors respectively. Each piece of silicon has nine pixels, that is nine individual sensors. The Low-energy spectrometer covers the electron energy range from $\sim 20 \mathrm{keV}$ to $\sim 240 \mathrm{keV}$ and a Medium-energy spectrometer covers the electron energy range from $\sim 80 \mathrm{keV}$ to $\sim 1200 \mathrm{keV}$. The configuration of the low and medium units is shown in Fig. 3 .

Representative electron trajectories are shown, with the higher-energy electrons striking further along the focal plane, where the pixels are of increased size. Protons are deflected away from the focal plane.

There are four pixels in a High-energy spectrometer rather than nine pixels as in the Low and Medium units. The front detector is a single piece of silicon, $0.3 \mathrm{~mm}$ thick. The rear detector is a sandwich consisting of three pairs of detectors, each pair consisting of two detectors each $1.5 \mathrm{~mm}$ thick. Thus there are six pieces of silicon in the stack, giving a total thickness of the rear detector of $9 \mathrm{~mm}$. The configuration of the high units is shown in Fig. 4 .

The two detectors for each pixel enable a coincidence measurement as a background reduction measure. The rear pairs are ganged together as needed to stop the maximum energy electrons that can reach a given pixel. Therefore Pixels 1 and 2 use only the first rear pair, Pixel 3 uses two of the pairs and Pixel 4 uses all three pairs. An unused pair, in the Pixel 1 stack, is used as a monitor of penetrating background. The front pixel has a smaller area than the back pixel and is centered upon the back pixel, reducing the percentage of electrons that make a valid coincidence but exit the thick rear detector out of a side rather than stopping. The energy coverage is from $800 \mathrm{keV}$ to $4800 \mathrm{keV}$.

\subsection{Magnet Design}

The MagEIS magnetic circuit designs are critical to a successful instrument. The MagEIS magnetic design uses magnetic materials, techniques, and design tools that have advanced considerably since the flight of the early Aerospace magnetic spectrometers. Advanced materials allow creation of the needed field strengths by smaller and lighter packages than possible before. These materials also permit precision magnetization techniques to be used to achieve tight tolerances on the resulting fields. Modern simulation tools allow rapid and accurate modeling of magnetic circuits to take much of the formerly needed guesswork out of the design process. 
Fig. 4 The High Spectrometer has a four-pixel focal plane consisting of four detector planes. The top plane is $0.3 \mathrm{~mm}$ thick and serves as a $\mathrm{dE} / \mathrm{dx}$ detector. The three rear planes, each $3 \mathrm{~mm}$ thick, serve as an $\mathrm{E}$ detector in a telescopic arrangement. The number of these planes used in a given pixel is selected according to the range (energy) of an electron that can reach a given pixel, from 1 to 3 going from Pixel 1 to Pixel 4. The unused rear pixels thus are colored red rather than green

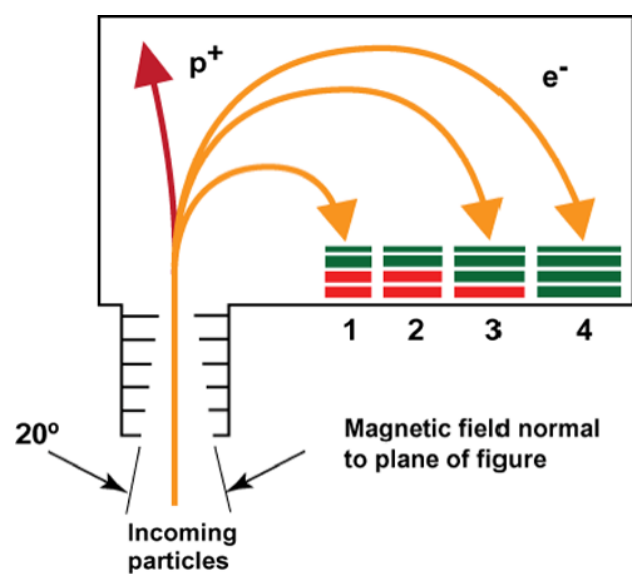

The geometric parameters of the MagEIS electron spectrometers are determined by a requirements flow, beginning with the desired energy coverage. The $180^{\circ}$ sector geometry specified for the magnets in practice is capable of covering only about one decade of dynamic range in energy (and less in the relativistic regime for an instrument like MagEISHigh) without putting undue constraints on other design parameters, such as size. Therefore MagEIS employs three magnetic chambers to cover the required energy range. The energy ranges for the chambers are thus based on this constraint. The required dynamic range in flux for each chamber is derived from empirically derived models of radiation belt fluxes, including a "worst-case" spectrum derived for the RBSP mission.

The flux-handling requirements then flow down to the geometric factor needed to have the required sensitivity while not being susceptible to saturation during large events. These considerations and design rules of thumb for magnetic spectrometers then establish the geometries for each of the chambers, which in turn produce the specification of the magnetic field in each. One of the design rules of thumb is that the detector pixels should be about the same size as the entrance aperture. Another rule of thumb is that the magnet gap should be kept short to constrain the out-of-plane angular acceptance, keep stray fields low, and make efficient use of magnetic energy. Keeping the gap short also helps keep the energy spread at a given radial detector position low, which is a key MagEIS concept in the pursuit of minimizing the amount of signal registered by penetrating background.

These requirements produce a common geometry for two of the chambers. The low/medium energy chambers have an entrance aperture of $2 \mathrm{~mm}$ by $5 \mathrm{~mm}$ and an angular acceptance of $20 \mathrm{deg}$ by $10 \mathrm{deg}$. Nominal field strengths for these designs are $550 \mathrm{G}$ and $1600 \mathrm{G}$, respectively, with a magnet gap of $7 \mathrm{~mm}$. The high chamber utilizes a larger entrance aperture $(10 \mathrm{~mm}$ high instead of $5 \mathrm{~mm})$ and has a field strength of $4800 \mathrm{G}$ with a gap of $12 \mathrm{~mm}$. The magnet gaps are larger than the aperture size in order to leave room for internal baffles that reduce scattering from the magnet pole faces. Figure 5 shows the design sensitivity for the three chambers, showing the one-count-per-spin-sector levels and the saturation levels for each.

An important constraint on the magnet chambers is the requirement that stray magnetic fields not interfere with the other RBSP instruments, in particular, the magnetometer. Static stray magnetic fields at the magnetometer location were to be less than a total of $5 \mathrm{nT}$. The resulting constraint is that the design and fabrication of the MagEIS magnet chambers must pay strict attention to the designs of the entrance and exit apertures and the yokes 


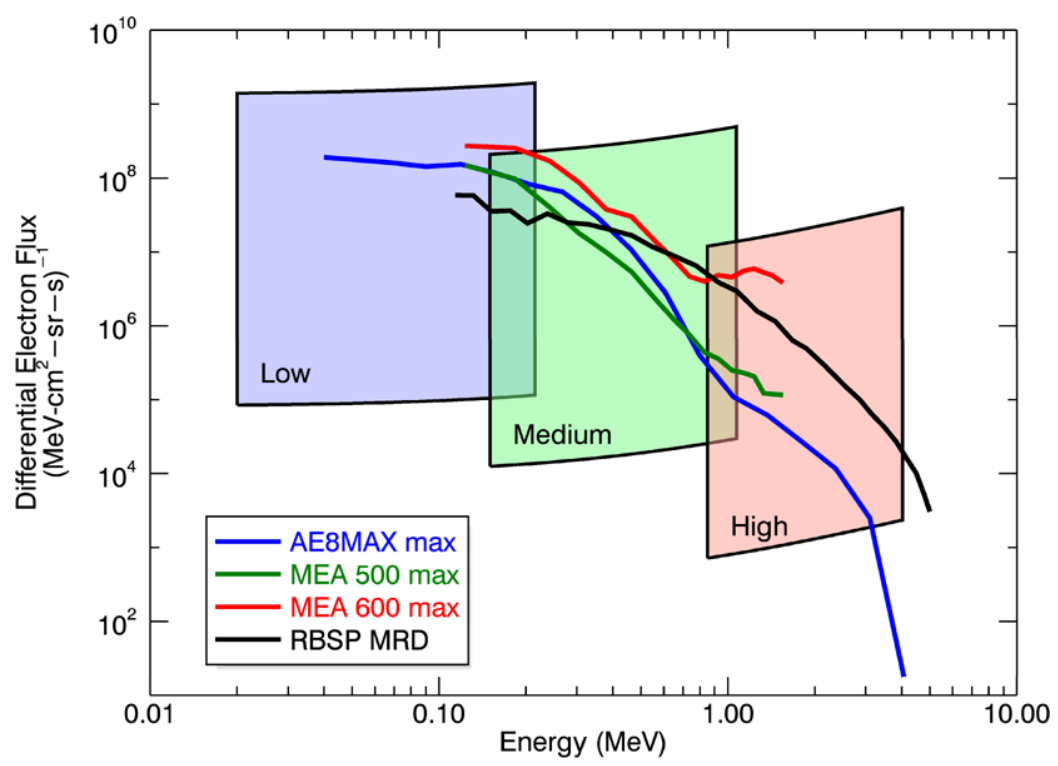

Fig. 5 Design sensitivity for the MagEIS electron instruments. The minimum and maximum are plotted for each of the three chambers based on one-count per sector ( $\mathrm{min}$ ) and electronics saturation (max). Also included for reference are the maximum fluxes that are expected based upon the AE8 radiation model, data from the CRRES mission (MEA), and the mission requirements for the RBSP. MEA 500 means CRRES Orbit 500, and MEA 600 means CRRES Orbit 600

used to close the flux path. A key aspect of the design is driven by this requirement; the detectors are enclosed within the magnet chambers, leaving only a small slit for electrical cabling. The stray field requirement also requires scrupulous attention to be paid to the way the chambers are assembled. Joining seams between yoke pieces are minimized, and the joining surfaces are lapped together before assembly in order to assure a magneticallytight assembly. Placing a shield of magnetically-soft material around the units was also contemplated as a way to reduce the challenge of creating low-leakage magnetic circuits. However, the polarizability of such shields created a larger problem than it solved, as the dynamic residual fields at the magnetometer that varied with spin phase, and RBSP has a more challenging requirement on dynamic stray fields (less than $0.1 \mathrm{nT}$ ). Thus MagEIS does not employ magnetic shields.

MagEIS uses high-performance magnetic materials. The magnets are made of highenergy product materials to enable small magnets to be used. In this way weight is kept relatively low, and, perhaps more importantly, packaging is kept small, keeping the requirements for yoke material low. The use of high-energy product magnets also allows for precise adjustment of the field in the gap, as the magnets are partially magnetized rather than using the maximum energy product available. This approach also allows a common geometry to be used for the low and medium chambers. For the magnets in these chambers a samariumcobalt (2-17) alloy having an energy product of 18 MGOe is used. A NdFeB alloy with energy product 50 MGOe provides the higher field strength required in the high chamber. In addition to these alloys having higher energy products than older materials such as Alnico, they also have higher intrinsic coercivity, making them easier to work with without their magnetization changing. The yoke material used in all chambers is Hiperco-50, a highcobalt steel alloy capable of carrying large flux densities without saturating. Unsaturated 


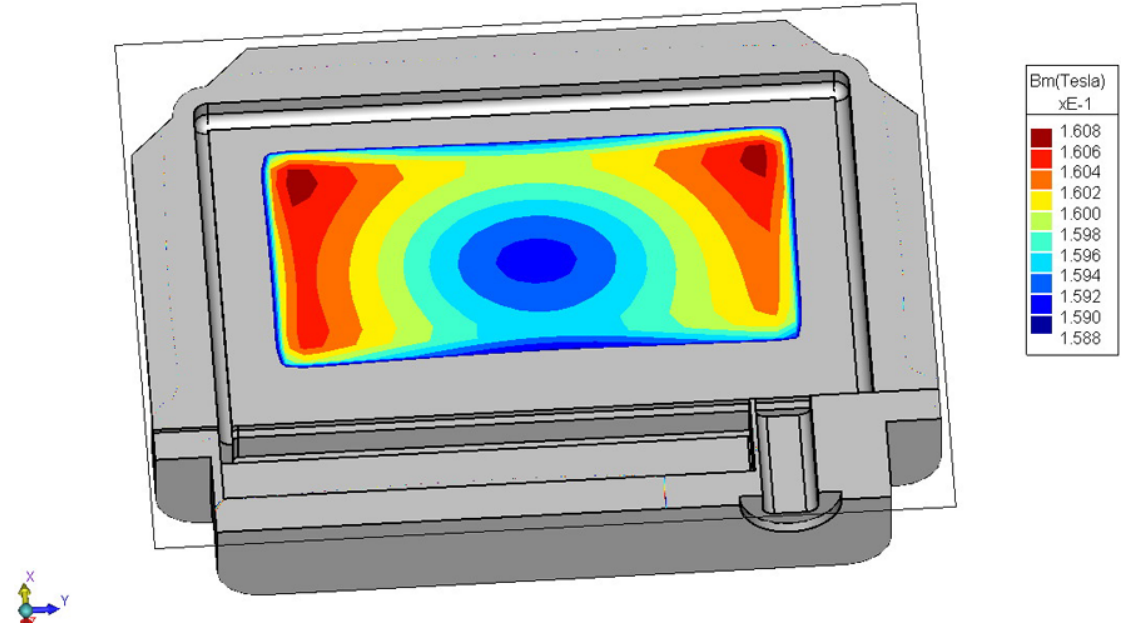

Fig. 6 Section view of the modeled magnetic circuits of the MagEIS medium electron chamber that has a mean field of $1600 \mathrm{G}$

yokes are important for keeping stray fields low, as well as allowing for efficient use of magnetic energy product.

A valuable product from the modeling is the good visualization of the field made possible, as shown in Fig. 6 for the medium chamber. The fields in all three chambers are quite uniform, with the low/medium chambers being within about $0.5 \%$ of the mean field over most of the volume, while the high chamber exhibits about $5 \%$ variation.

The performance of the magnetic chambers also was simulated by tracing particle trajectories through the modeled magnetic systems. The commercial product SIMION 8.0.4 was employed for this purpose due to its ability to compute the trajectories accurately and rapidly. The B-fields were read in from the Lorentz simulation. Note that the simulation does not include the effects of scattering. Figure 7 is an example of tracing some electron trajectories through the medium unit. It illustrates the first-order focus of particles with the same energy. The focusing is not quite ideal, likely owing to the effects of non-idealities in the magnetic field.

More detailed estimates of the instrument performance were obtained by launching large numbers of particles in such a manner for each chamber. A Monte Carlo scheme was used to sample the full gamut of particle trajectory parameters to examine the performance. An example appears in Fig. 8, which shows the distribution of detected energies and angles for Pixel 5 of the detector in the low chamber given an input beam that completely fills the collimator and has a flat energy spectrum. It shows the aggregate effect of the non-ideal field, as the energy-angle mixing should appear as a "smile" for perfect first-order focusing. Instead, only a portion of the "smile" appears. However, the amount of distortion is small enough that MagEIS can still meet its requirements. These simulations also provide accurate estimates for the magnetic chamber performance parameters listed in Table 1.

\subsection{Further Design Considerations-Energy Histograms}

In order to determine and correct for background in the silicon detectors, we measure the entire pulse-height spectrum in each detector from just above the noise level to amplifier sat- 
Fig. 7 Illustration of particle trajectory tracing in the modeled magnetic field for the medium chamber. Depicted is a cross-section of the geometry, with $800-\mathrm{keV}$ electrons incident from the left

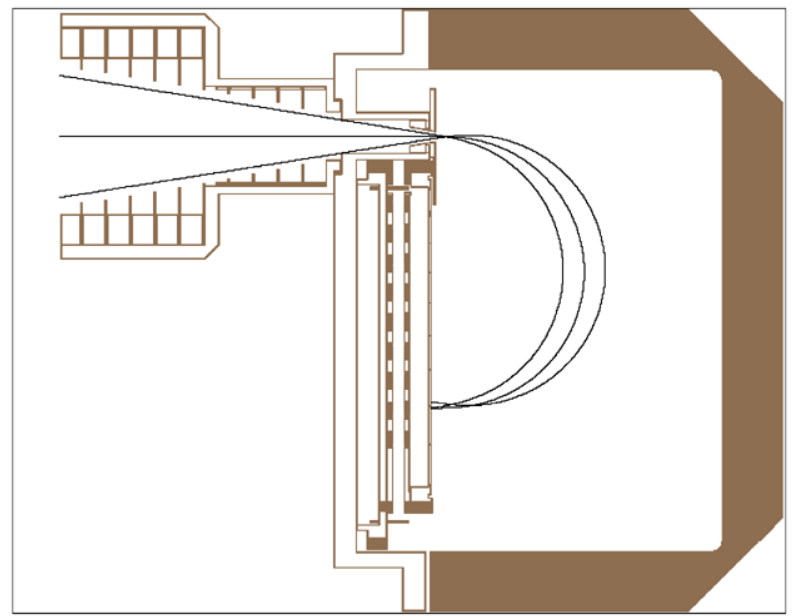

Fig. 8 The distribution of detected energies and angles for Pixel 5 of the detector in the low chamber is plotted

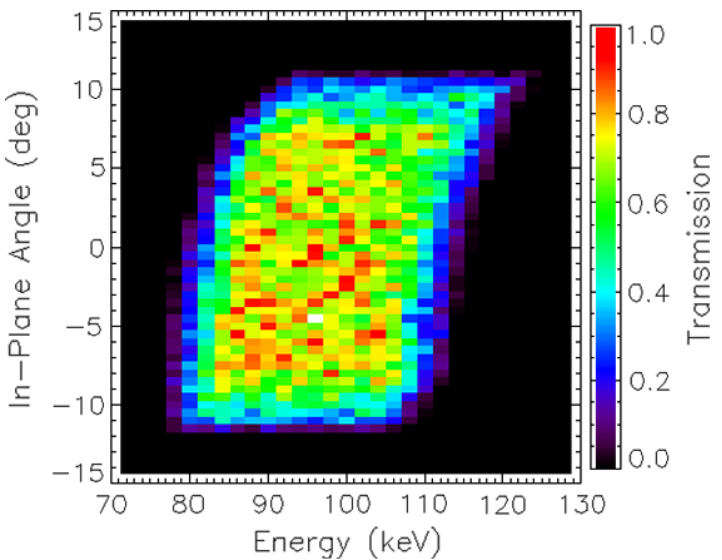

uration, not just those events within the energy pass band of a given detector, cf. Fig. 9. The count rate peak, appropriate to the detector in question, is seen "riding" on a smooth background spectrum, which is largely due to bremsstrahlung from the intense electron fluxes striking the RBSP spacecraft. In those regions of space where energetic trapped protons are found (the inner zone), and during energetic solar particle events, large pulses will result from ions that penetrate the magnetic spectrometer and reach the focal plane detectors. The key point is that with this technique, background events can be unequivocally determined and subtracted from the count rate within the differential energy channel of each detector, giving the true count rate in each channel. Furthermore, should the background swamp the true count rate, this fact will be obvious.

On-orbit data is shown to supplement the idealized discussion of signal and background given above.

In Fig. 10 the pass band of Pixel 4 is clearly seen when the satellite is at $L^{*}=5.6$ (red curve) as a nearly rectangular increase in count rate between channel 24 and channel 44 . The flat-topped region between channel 27 and channel 38 are the prime data region for Pixel 4 as discussed below. A first order correction for the background can be made by smoothly joining the count rate curve below channel 23 to the section above channel 45 , 
Table 1 Estimated values for several MagEIS performance parameters derived by simulating particle trajectories through the model magnetic field for each chamber. Values for each detector pixel are shown. Actual telemetered channels do not sum over all PHA energy channels for each pixel and therefore have different effective geometric factors. Energy widths and angular acceptances are full-width-at-half-maximum

\begin{tabular}{|c|c|c|c|c|c|c|}
\hline Chamber & Pixel & $\begin{array}{l}\text { Median } \\
\text { energy } \\
(\mathrm{keV})\end{array}$ & $\begin{array}{l}\text { Energy } \\
\text { width } \\
(\mathrm{keV})\end{array}$ & $\begin{array}{l}\text { Geometric } \\
\text { factor } \\
\left(\mathrm{cm}^{2} \mathrm{sr} \mathrm{keV}\right)\end{array}$ & $\begin{array}{l}\text { In-plane } \\
\text { angular } \\
\text { acceptance } \\
\text { (deg) }\end{array}$ & $\begin{array}{l}\text { Out-of-plane } \\
\text { angular } \\
\text { acceptance } \\
\text { (deg) }\end{array}$ \\
\hline \multirow[t]{8}{*}{ Low } & 1 & 19.5 & 10.6 & 0.0414 & 20 & 9.8 \\
\hline & 2 & 32.7 & 14.3 & 0.0526 & 20 & 7.8 \\
\hline & 3 & 50.0 & 18.6 & 0.0607 & 20 & 6.4 \\
\hline & 4 & 71.8 & 23.0 & 0.0663 & 20 & 5.6 \\
\hline & 5 & 98.1 & 27.3 & 0.0703 & 20 & 4.7 \\
\hline & 6 & 129 & 32.2 & 0.0716 & 20 & 4.0 \\
\hline & 7 & 166 & 37.3 & 0.0724 & 20 & 3.6 \\
\hline & 8 & 207 & 42.7 & 0.0726 & 20 & 3.2 \\
\hline \multirow[t]{8}{*}{ Medium } & 1 & 146 & 72.4 & 0.281 & 20 & 10 \\
\hline & 2 & 232 & 89.7 & 0.328 & 20 & 7.9 \\
\hline & 3 & 334 & 106 & 0.342 & 20 & 6.8 \\
\hline & 4 & 450 & 121 & 0.343 & 20 & 5.8 \\
\hline & 5 & 580 & 132 & 0.332 & 20 & 4.7 \\
\hline & 6 & 722 & 145 & 0.316 & 20 & 4.1 \\
\hline & 7 & 877 & 151 & 0.303 & 20 & 3.4 \\
\hline & 8 & 1040 & 163 & 0.287 & 20 & 3.1 \\
\hline \multirow[t]{4}{*}{ High front } & 1 & 1200 & 452 & 4.53 & 16 & 19 \\
\hline & 2 & 1740 & 452 & 4.56 & 16 & 19 \\
\hline & 3 & 2520 & 896 & 8.38 & 16 & 16 \\
\hline & 4 & 3770 & 1170 & 8.26 & 16 & 11 \\
\hline \multirow[t]{4}{*}{ High back } & 1 & 1220 & 489 & 4.89 & 16 & 19 \\
\hline & 2 & 1740 & 516 & 5.28 & 16 & 19 \\
\hline & 3 & 2510 & 1030 & 9.65 & 16 & 16 \\
\hline & 4 & 3750 & 1400 & 9.57 & 16 & 11 \\
\hline
\end{tabular}

and subtracting the values thus obtained from the count rate in the pass band. In this case the background is no more than $10 \%$.

The background when the spacecraft is at $L^{*}=3.5$ can be seen to completely mask any true signal. At $L^{*}=2.0$ counts in the pass band lie well above the background. The counts within the pass band drop off markedly within the pass band rather than having a flat top because the energy spectrum is much softer than at $L^{*}=5.6$.

Finally at $L^{*}=1.3$ the background is large but the true signal can still be seen riding on this background. These data are spin averaged because no magnetic aspect data are available at the time of writing, five months after launch.

The power of the background subtraction system can be further seen by examining the response in a higher energy pixel in the same spectrometer, Fig. 11 data are taken at the same times as in Fig. 10. 
Fig. 9 A valid signal is shown schematically as combined with penetrating background events in a given pixel pass band
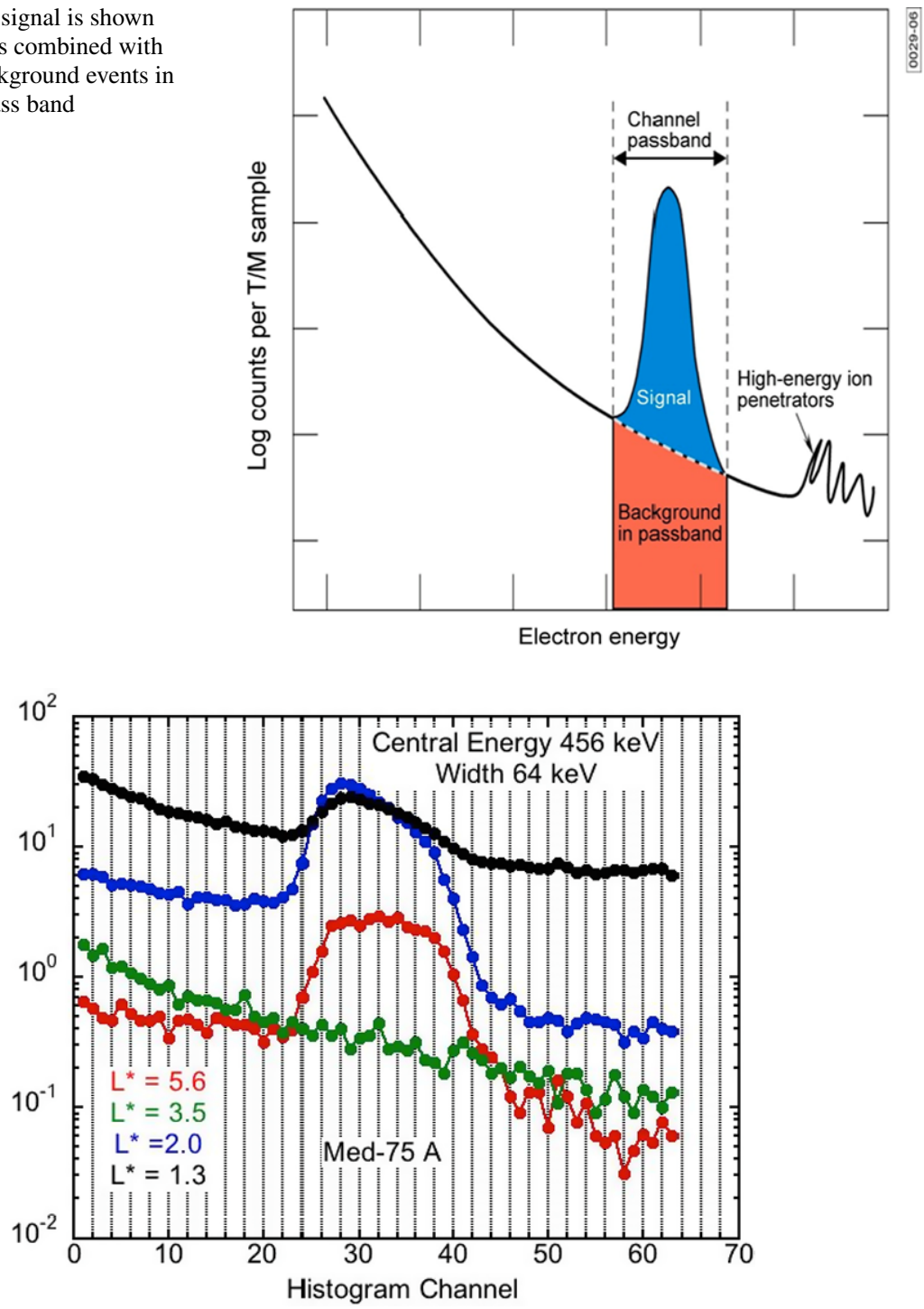

Fig. 10 The histogram data from Pixel 4 of the Med-75 unit of spacecraft A is shown for four values of $L^{*}$. Data were acquired on 25 December 2012

Electrons can clearly be seen above the background at $L^{*}=5.6$ although the intensity is much lower than in Pixel 4 shown in Fig. 10. However there is no signal in Pixel 7 at the other three values of $L^{*}$. This result is in marked contrast to the situation for Pixel 4.

The MagEIS analysis system is even more robust against background than simply as described above. Focal plane detectors are adjacent, and the background energy spectrum will be much the same in adjacent detectors. Deviation from this expectation will provide a direct warning that something is amiss. The superiority of a magnetic spectrometer of this type is clear; not only is a two-parameter analysis made for every electron but the entire gamut of background events is measured as well. 


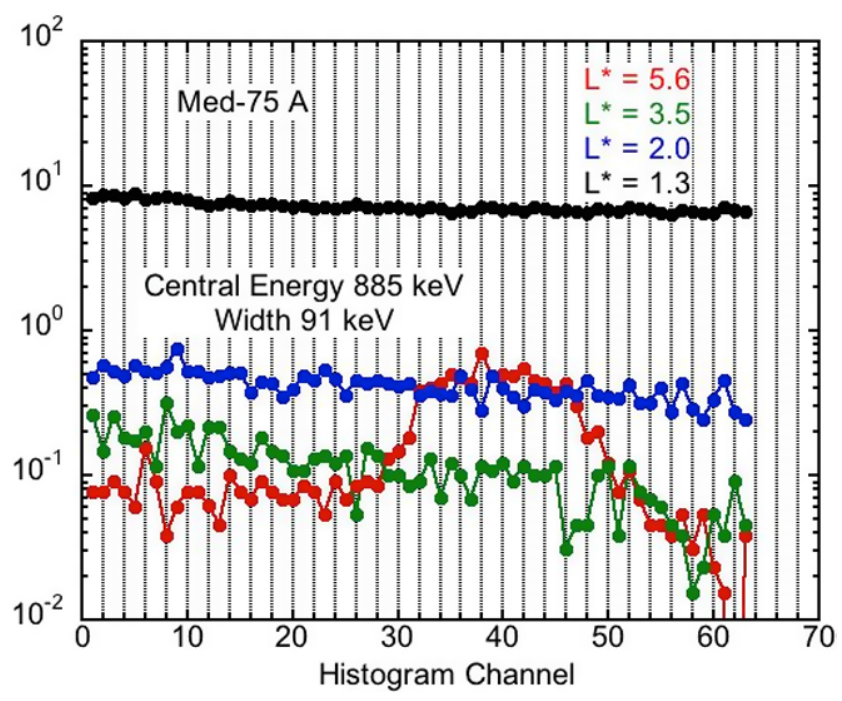

Fig. 11 The histogram data from Pixel 7 of the Med-75 unit of spacecraft 4A is shown for four values of $L^{*}$. Data were acquired on 25 December 2012

\subsection{Multiple Fields of View}

The entrance aperture of each magnetic spectrometer is rectangular, with an opening angle of 10 degrees in the azimuth direction and 20 degrees in the polar direction and the spacecraft rotation provides a pitch-angle scan in the usual manner. There are one low-energy, one medium-energy, and one high-energy spectrometer pointing away from the spacecraft close to the nominal bellyband direction (i.e. close to the direction perpendicular to the S/C spin axis). The field-of-view of each of these sensors is offset by 15 degrees from a perpendicular direction in order to increase the range of observable pitch angles.

In addition, a second medium-energy spectrometer is mounted with a look-direction centered at 35 degrees with respect to the $\mathrm{S} / \mathrm{C}$ spin axis in order to increase pitch angle coverage during those time periods when the ambient magnetic field is stretched and far from dipolar.

A number of pointing configurations were examined in order to optimize pitch-angle coverage from the two look directions given by the medium energy MagEIS instruments. Since the spin axis of the spacecraft is designed to point approximately toward the sun, small values of beta, the angle between the magnetic field and the spin axis, are indicative of highly stretched field configurations on the day side and night side, while large values of beta result from more dipolar field configurations. On the dusk and dawn sides, stretched fields do not necessarily imply small values of beta because the field direction is typically more perpendicular to the spin axis there. However, very stretched configurations often exist during storm conditions, which is a principal science target for the mission. To ensure reasonable coverage during such events, the detectors were oriented at $75 \mathrm{deg}$ and $35 \mathrm{deg}$ (each with 20 degree field of view). For such a configuration, good pitch angle coverage is maintained for values of beta as low as $10 \mathrm{deg}$.

Figure 12 shows the pitch angle coverage (and overlap) achieved by the two Medium MagEIS instruments. In the plot, the vertical axis gives the pitch angle (alpha), and the horizontal axis is beta. 
Fig. 12 The pitch-angle coverage of the two Medium MagEIS spectrometers

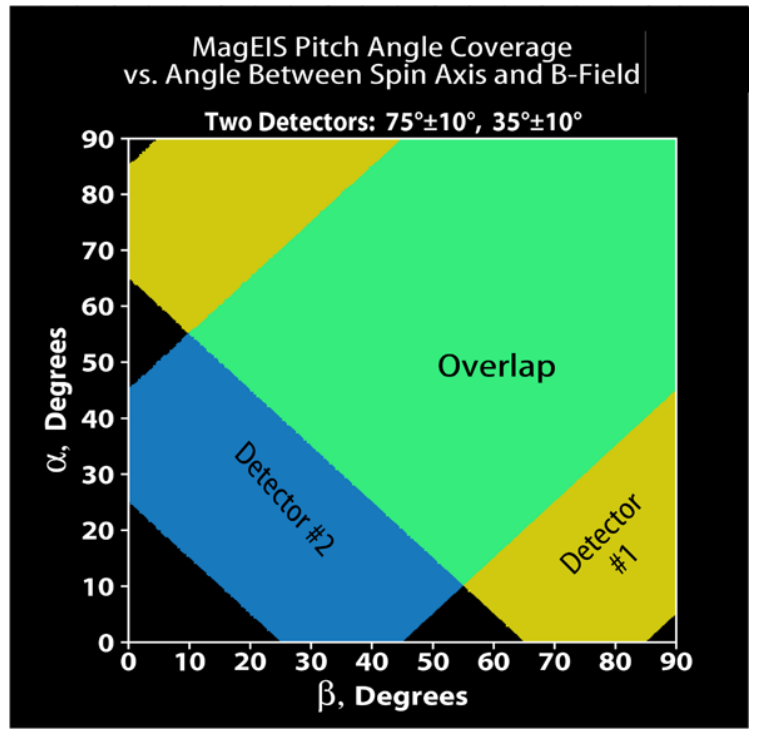

The coverage in the alpha-beta parameter space from each detector is colored-coded. Blue shows regions covered by the more spin-axis aligned detector (MagEIS-Med35) alone, while yellow shows regions covered by the other detector (MagEIS-Med75) alone. Green shows regions of overlapping coverage.

\section{Instrument Design-Ions}

\subsection{Overview}

In addition to a magnetic electron spectrometer, both MagEIS-HI instruments contain a silicon-detector telescope. The first detector in both telescopes measures the fluxes, energy spectra, and angular distributions of protons in the energy range from $50 \mathrm{keV}$ to greater than $1 \mathrm{MeV}$. These telescopes have no composition discrimination but protons of course will dominate; they are identical in RBSP A and RBSP B.

Two additional detectors follow in both telescopes but are not identical; the configuration differences and measurements made by these detectors are discussed below.

\subsection{Low Energy Protons}

The proton telescope mechanical configuration is shown in Fig. 13. The front detector is a Micron Semiconductor device that is $\mathbf{5 0 ~ m i c r o n s ~ t h i c k , ~ a n d ~ i s ~ t o t a l l y ~ d e p l e t e d . ~ T h e ~ o u t s i d e ~}$ diameter of the sensitive area is $8 \mathrm{~mm}$ and the inner annulus is $4 \mathrm{~mm}$ in diameter. Particles that pass through the annulus strike the rear detectors; the configurations and functions of the rear detectors are discussed below. The detectors are positioned behind a disk-loaded collimation system with a 1.5-kilogauss electron-sweeping magnet in the middle of the collimator. Laboratory testing has shown that this electron sweeper prevents electrons below $\sim 2 \mathrm{MeV}$ from striking the proton detector and higher energy electrons are largely attenuated.

There are nominally 20 rate (energy) channels, sampled 20 times per spacecraft rotation from the front detector. There are histogram data from both the front and rear detectors. 
MagEIS High - Proton Telescope

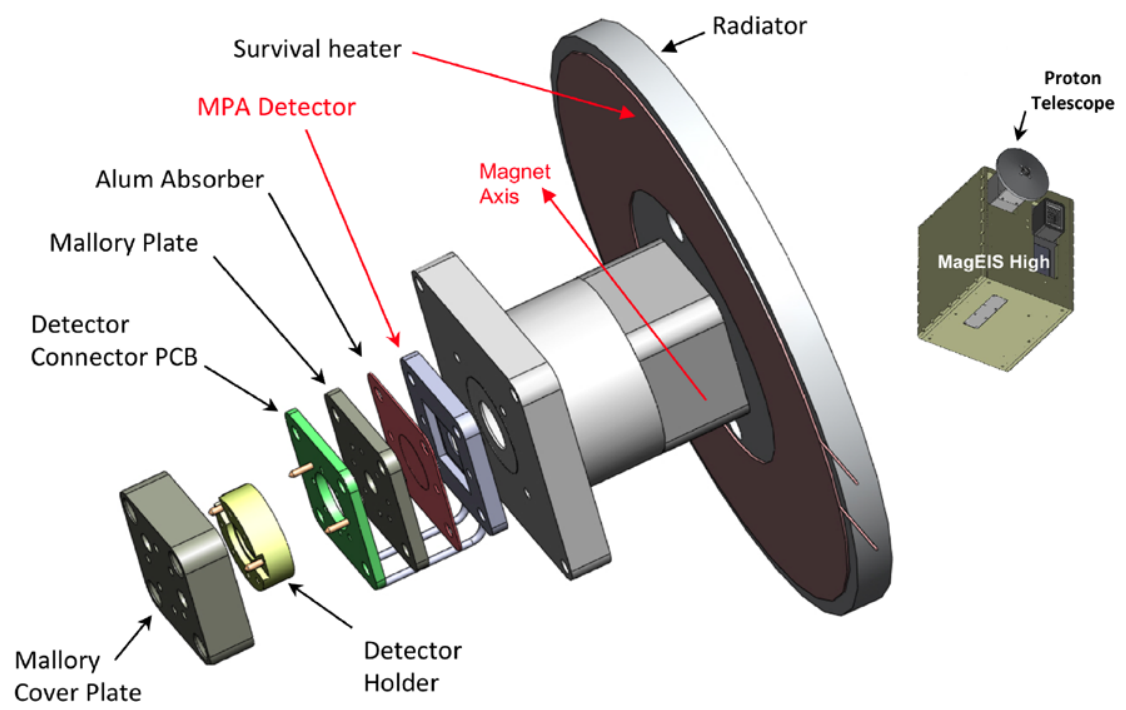

Fig. 13 An exploded view of the mechanical configuration of the proton telescope
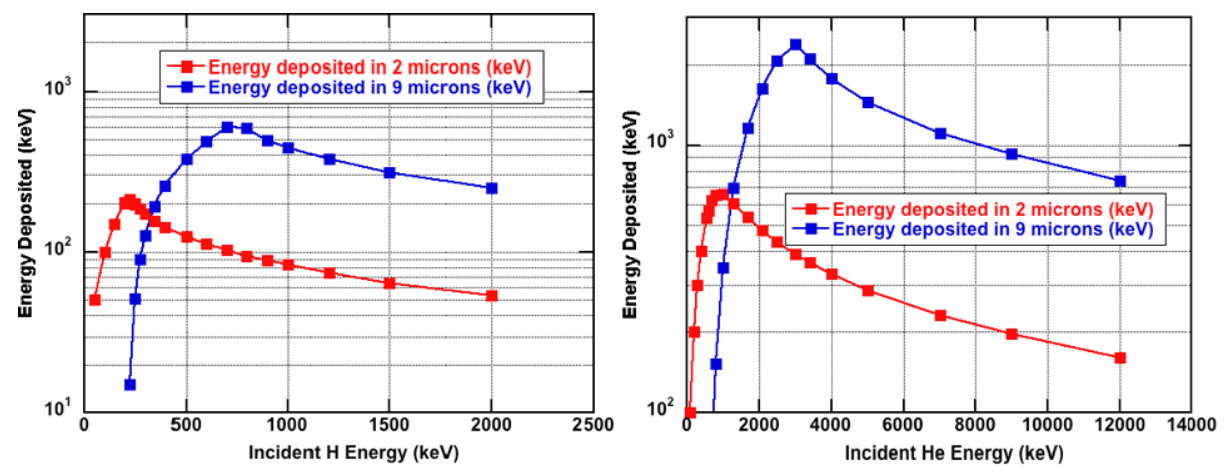

Fig. 14 The figures give the energy deposits in the 2 micron and 9 micron detectors by $\mathrm{H}$ and $\mathrm{He}$ ions as a function of incident ion energy, in the left and right panels respectively

\subsection{Heavy Ions (Telescope in RBSP A-Proton Telescope A)}

Two ORTEC surface-barrier detectors are placed inline, and view space through a $3.8 \mathrm{~mm}$ diameter collimator placed just behind the $4 \mathrm{~mm}$ hole in the center of the front annular proton detector. A 2-micron detector is in front and a 9-micron detector behind. Thus ions striking the 9-micron detector must first pass through the 2-micron detector. There is no coincidence formed between events in these two detectors.

The SRIM 2011 software was used to calculate the energy deposit of $\mathrm{H}, \mathrm{He}$, and $\mathrm{O}$ ions over the energy ranges of interest, results are shown in Fig. 14. 
Table 2 Helium Energy Channels in RBSP A Telescope

Fig. 15 The calculated response of the 2 micron and 9 micron detectors to $\mathrm{O}$ ions is shown

\begin{tabular}{lll}
\hline He2L & $350 \mathrm{keV}$ & $350 \mathrm{keV}-3500 \mathrm{keV}$ \\
$\mathrm{He} 2 \mathrm{H}$ & $500 \mathrm{keV}$ & $500 \mathrm{keV}-2000 \mathrm{keV}$ \\
$\mathrm{He} 9 \mathrm{~L}$ & $900 \mathrm{keV}$ & $1500 \mathrm{keV}-9500 \mathrm{keV}$ \\
$\mathrm{He} 9 \mathrm{H}$ & $1500 \mathrm{keV}$ & $2000 \mathrm{keV}-4800 \mathrm{keV}$ \\
\hline
\end{tabular}

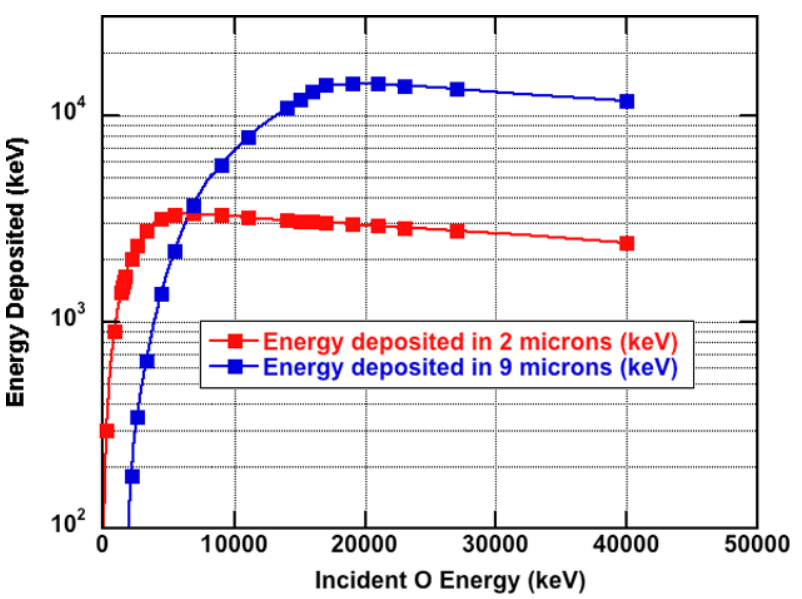

The selected energy thresholds render these detectors insensitive to protons. Each of the two detectors has two energy deposit thresholds; He2L(low); He2H(High); He9L(low); $\mathrm{He} 9 \mathrm{H}$ (high). The thresholds and sensitive energy ranges for He ions are given in Table 2.

These two rate (energy) channels, are sampled 20 times per spacecraft rotation in an analogous fashion to the proton data from the front detector.

Oxygen is another ion of high scientific interest for the RBSP mission. Figure 14 shows that $\mathrm{He}$ cannot deposit more than $\sim 700 \mathrm{keV}$ in the 2-micron detector and more than $\sim 2200 \mathrm{keV}$ in the 9-micron detector. Trapped ions depositing higher energies must be heavier than $\mathrm{He}$, and are expected in general to be due to $\mathrm{O}$ ions in the Earth's magnetosphere. An energy deposition plot for $\mathrm{O}$, similar to the two above for $\mathrm{H}$ and for $\mathrm{He}$, is shown in Fig. 15.

Because of the expected low rate of oxygen events, there are no rate channels. Histogram data are acquired from both detectors, 64 channels from each detector covering the energy ranges from $400 \mathrm{keV}$ to $4 \mathrm{MeV}$ for the 2-micron detector, and $900 \mathrm{keV}$ to $22 \mathrm{MeV}$ for the 9-micron detector. The histograms are nominally read out every eight spacecraft rotations.

Although the energy/species covered by these two detectors are described by the SRIM software, gains and energy responses were measured using several heavy-ion beams accelerated by the LBNL $88^{\prime \prime}$ cyclotron.

\subsection{Energetic Protons and Heavy Ions (Telescope in RBSP B-Proton Telescope B)}

Two Micron Semiconductor ion-implanted detectors are stacked inline, and view space through a $3.8 \mathrm{~mm}$ diameter collimator placed just beyond the $4 \mathrm{~mm}$ hole in the center of the annular proton detector.

The 10-micron detector is used to make heavy-ion measurements much as done with the two thin detectors in the proton telescope in RBSP A. This detector also serves to block 
Fig. 16 The calculated response of the telescope to energetic protons is shown
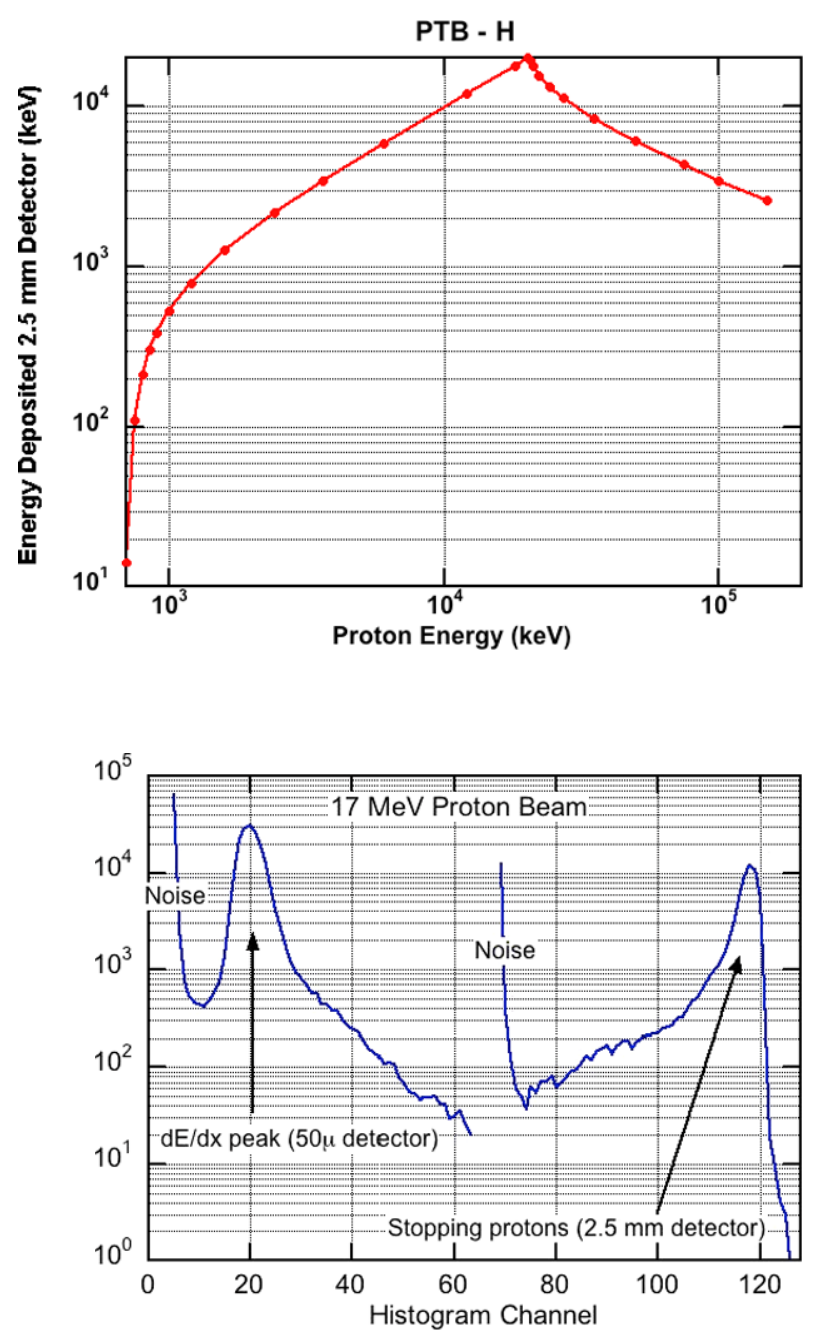

Fig. 17 The response of Proton Telescope B to a $17 \mathrm{MeV}$ proton beam is shown for both the $50 \mu$ and the $2.5 \mathrm{~mm}$ detectors. The beam is stopped in the thicker detector giving a total energy peak whereas the $50 \mu$ detector gives a clean $\mathrm{dE} / \mathrm{dx}$ peak made by the penetrating protons

low-energy protons from being incident upon the 2500-micron detector and thus eliminates a high event rate below the $\mathrm{MeV}$ energy range of interest.

The primary function of the 2500-micron detector is to fill the energy gap between $\sim 800 \mathrm{keV}$ and $20 \mathrm{MeV}$, the latter energy being near the proton threshold of the REPT instrument in the ECT suite. Figure 16 shows the energy deposited in the 2500-micron detector as a function of the incident proton energy.

Near the low-energy threshold, this thick detector augments the measurements made by the primary (front) detectors in both PTA and PTB as can be seen in the following plot. Each detector has a low and a high threshold rate channel. The rate channels are sampled $\sim 20$ times per spacecraft rotation. Each detector has a histogram output. The histograms are read out nominally once every 8 spacecraft rotations.

The sensor was calibrated using proton beams between 6 and $50 \mathrm{MeV}$ accelerated by the LBNL 88" cyclotron. An example of such calibration data is shown in Fig. 17. 

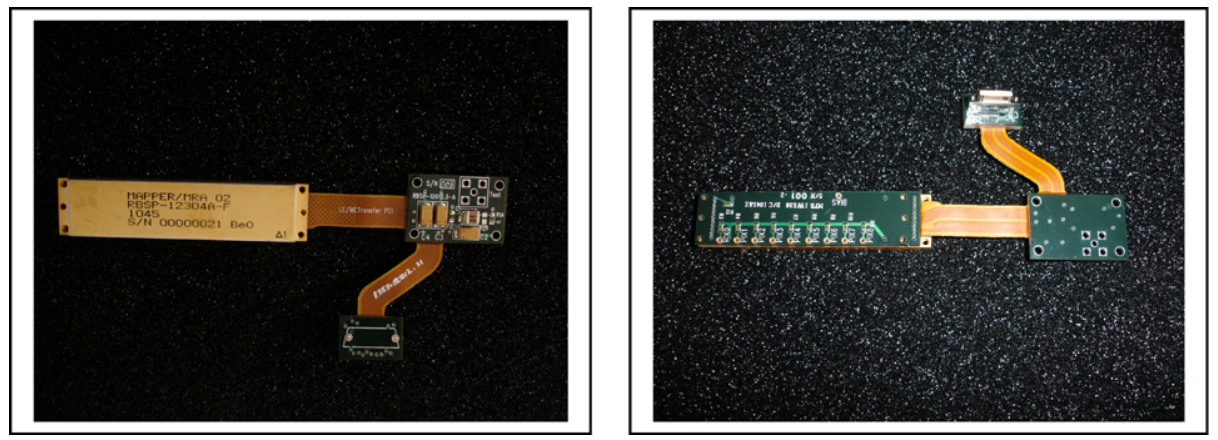

Fig. 18 The MAPPER hybrid attached to Transfer PCB is shown in the left panel. The silicon detector array attaches to the sockets on the side of the PCB opposite the MAPPER, right panel

\section{MagEIS Front-End Electronics}

\subsection{Electronic Design and Configuration}

Each of the MagEIS instruments uses a custom multi-chip module (i.e., hybrid) to amplify and digitize the charge pulse from the silicon detector pixels in the electron spectrometer and proton telescope. The MAPPER hybrid (Multi-Amplifier Pulse Peak Energy Readout) provides an architecture that offers parallel analog processing and digital readout for ten detector pixels. The hybrid is designed to attach to the backside of a detector array with minimal interconnect length, thus minimizing the noise contribution of parasitic capacitance. A rigid-flex circuit board, called the Transfer PCB, is used to provide the detector connection as well as the interface to the digital processing unit (DPU) as shown in Fig. 18.

The analog MAPPER chip and digital MRA chip together form a complete pulse-height analysis (PHA) system. Figure 19 shows the functional block diagram of the hybrid electronics. Each MAPPER amplifier channel contains a charge preamplifier, pole-zero cancellation network, shaping amplifier, scaling amplifier, baseline restorer, and peak-hold amplifier. The front-end is AC coupled to the detector and measures positive charge flowing into the preamplifier feedback capacitance, which can be set to $0.2 \mathrm{pF}$ or $1 \mathrm{pF}$ depending on the gain selection. The preamplifier is continuously reset through a linear-mode FET in the feedback loop. The shaping amplifier produces a 3-pole semi-Gaussian pulse with shaping time of $\sim 1$ usec FWHM. This signal is then scaled by another amplifier such that the full-range is approximately 1-volt.

The energy deposited in the detector is linearly proportional to the peak of the shaped detector pulse. A discriminator is used to determine if the peak of the shaped pulse exceeds a pre-stored threshold charge on the peak-hold capacitor. When this happens, a digital peak-detect signal is asserted to indicate that the shaped pulse peak is stored and ready for conversion by the MRA (MAPPER Readout ASIC).

A Wilkinson A/D converter was chosen for its high linearity and low power dissipation, making it suitable for dedicated use on each amplifier channel. The Wilkinson A/D is formed by the way the peak-hold capacitor is discharged. When the peak of the shaped pulse is stored and the peak-detect signal goes active, a constant current source inside the MAPPER chip is activated by the MRA. The current source is pre-tuned by an internal resistor on each MAPPER channel, called the rundown resistor, to give a constant linear decay rate. A buffered form of the peak-hold capacitor voltage is presented to a comparator, which 


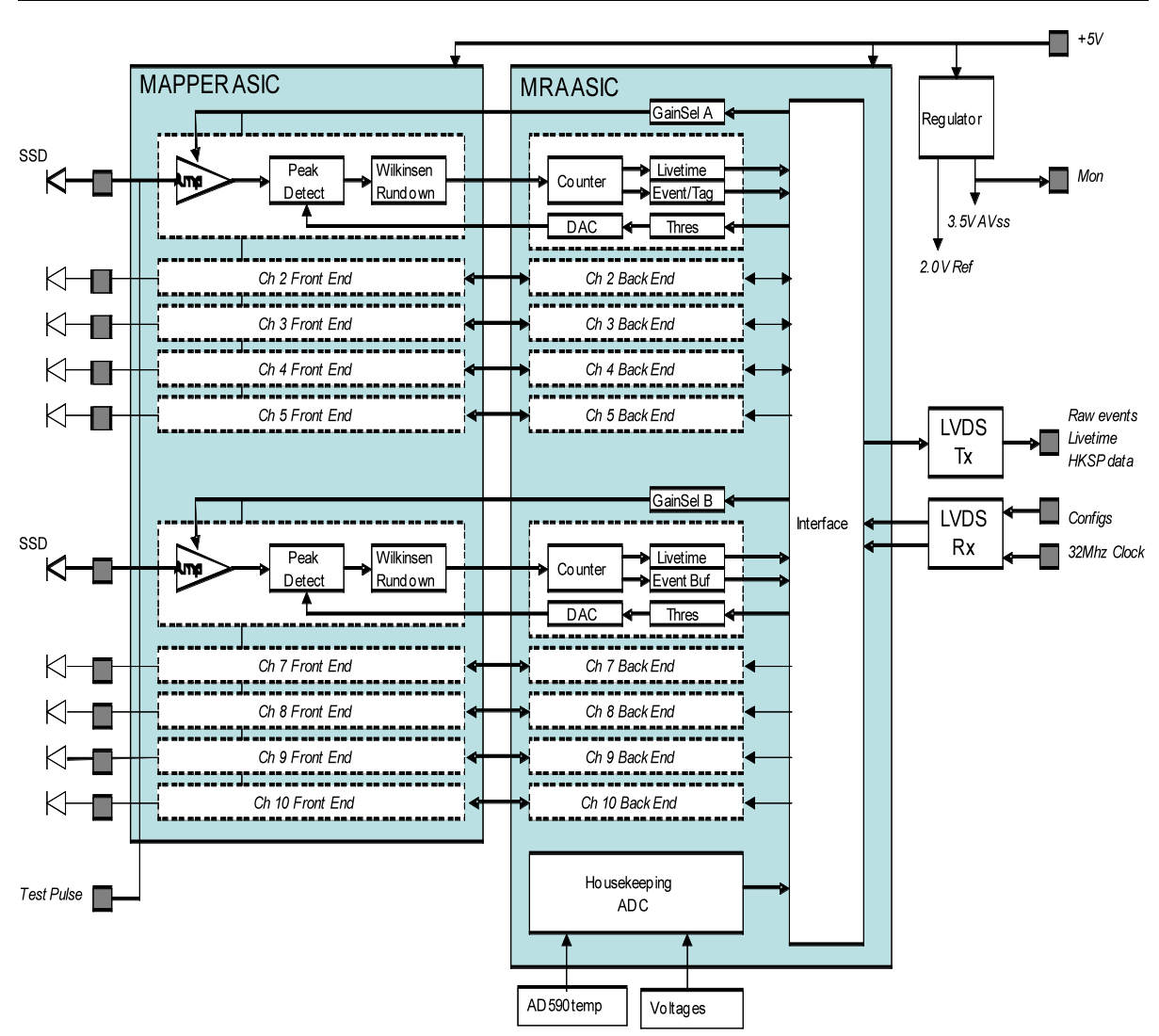

Fig. 19 MAPPER dual-ASIC hybrid functional block diagram

goes active when it drops below the baseline reference, marking the end of the rundown interval and the de-assertion of the peak-detect signal. The width of the peak-detect signal is proportional to the peak of the shaped pulse, and hence the energy deposited in the detector. The MRA chip digitizes this width by counting the number of $32 \mathrm{MHz}$ system clocks between the assertion and de-assertion of the peak-detect signal. Although a much larger dynamic range could be achieved with this $\mathrm{A} / \mathrm{D}$ converter, only 8-bits are needed to meet the measurement requirements.

The MAPPER hybrid is designed with flexibility to support all the detector requirements. By selecting one of several gain modes (i.e., range modes), the hybrid can be optimized for different energy ranges depending on the targeted instrument. Table 3 gives the nominal energy ranges selected for each of the MagEIS instrument types. In addition, the MRA chip provides a dedicated programmable 12-bit D/A threshold value for each MAPPER channel.

A single MAPPER can also be configured to support two different detectors. This was necessary for the High instrument electron detectors, whereby the front detector and back detector arrays have different required energy ranges. The MAPPER is organized into two banks, each containing five amplifier channels, which share a common range setting.

The MRA chip provides important timing information. Each direct-event is time-tagged and appended to the PHA word. This time-tag is 8-bits and has a resolution of 1-usec. It is used by the DPU to determine coincident events in the High unit detector stack. The MRA 
Table 3 MAPPER energy range settings

\begin{tabular}{lll}
\hline $\begin{array}{l}\text { MAPPER Range } \\
\text { Setting }\end{array}$ & $\begin{array}{l}\text { Full-scale } \\
\text { Energy }\end{array}$ & Detectorv System \\
\hline Low Range & $280 \mathrm{keV}$ & Low Electron Array \\
Medium Range & $1.25 \mathrm{MeV}$ & $\begin{array}{l}\text { Medium Electron Array, } \\
\text { High Front Electron Array } \\
\text { High Back Electron Array }\end{array}$ \\
High Range & $4.5 \mathrm{MeV}$ & \\
\hline
\end{tabular}

also provides 16-bit live-time counters for each channel, having a resolution of $32 \mathrm{u}$-secs for monitoring the amount of time the channel is not processing an event (i.e., the opposite of dead-time).

The MAPPER hybrid provides some diagnostic capability. A test pulser input is connected in common to each of the preamplifiers through an on-chip injection capacitor. The DPU activates the pulser during instrument checkout to verify that the gain, offset, and noise of each channel is within specifications. The MAPPER hybrid also provides an 8-bit successive-approximation A/D converter for monitoring the internal temperature, reference voltage, preamplifier source voltage, and baseline levels for both banks.

Bi-directional communication between the MAPPER hybrid and the DPU is established by a synchronous LVDS (Low Voltage Differential Signaling) interface. Direct event data, live-time data, and housekeeping are transmitted to the DPU by a LVS transmitter chip, while configuration commands are received by a LVDS receiver chip, both of which are internal to the hybrid. LVDS was chosen to minimize digital coupling to the front-end. This choice allowed simultaneous operation of the front-end with digital transmission of events, thus eliminating the dead-time that is encountered by systems that disable the front-end during data transfer. The LVDS operates from a $32 \mathrm{MHz}$ clock, which is also driven as an LVDS signal to minimize noise

\subsection{Engineering Calibrations-Gain/Offset Measurements}

The gains and offsets of the electronic chains of every pixel were measured over the operational temperature range as well as at room temperature. Every low and medium magnetic spectrometer has a 9 pixel focal plane for a grand total of 54. A high-energy unit has 8 pixels in two planes and each proton telescope in both high units contains 3 detectors. Thus the total number of detectors is 76 in the MagEIS complement. Since gain/offsets of every detector were measured at a least three temperatures, often more, the total number of individual gain/offset measurements made in preparing the eight spectrometers for flight was well over two hundred. Here we will show a few examples and some summaries that are representative of the entire database. Figure 20 is a calibration example that employed a radioactive source.

The Bi-207 source emits electrons at several discrete energies resulting in distinct peaks over the energy range of the focal plane. The channel location was accurately determined by fitting every peak to a Gaussian in channel space. The energies of the various electrons are well known and the peak location can then be plotted vs. peak energy as shown in Fig. 21.

It can be seen that a linear fit is excellent, showing that the electronics have good linearity. The offset of $-17.7 \mathrm{keV}$ indicates that zero energy is not at channel 0 . Figures 22 and 23 present a summary of the gains and offsets for the nine pixels in the medium energy spectrometer as a function of temperature.

The two plots show that the temperature dependence of both the gains and offsets is small, especially the case of the gains. Furthermore the pixel-to-pixel variation in gain is small also. 
Fig. 20 A plot of the electron energy spectrum of $\mathrm{Bi}-207$ as seen in Pixel 1 of FM301 at $-8 \mathrm{C}$ is shown

Fig. 21 The channel locations of the energy peaks plotted in Fig. 20 is plotted vs. the energy of the respective peak. The resulting energy vs. channel plot is fitted to a straight line
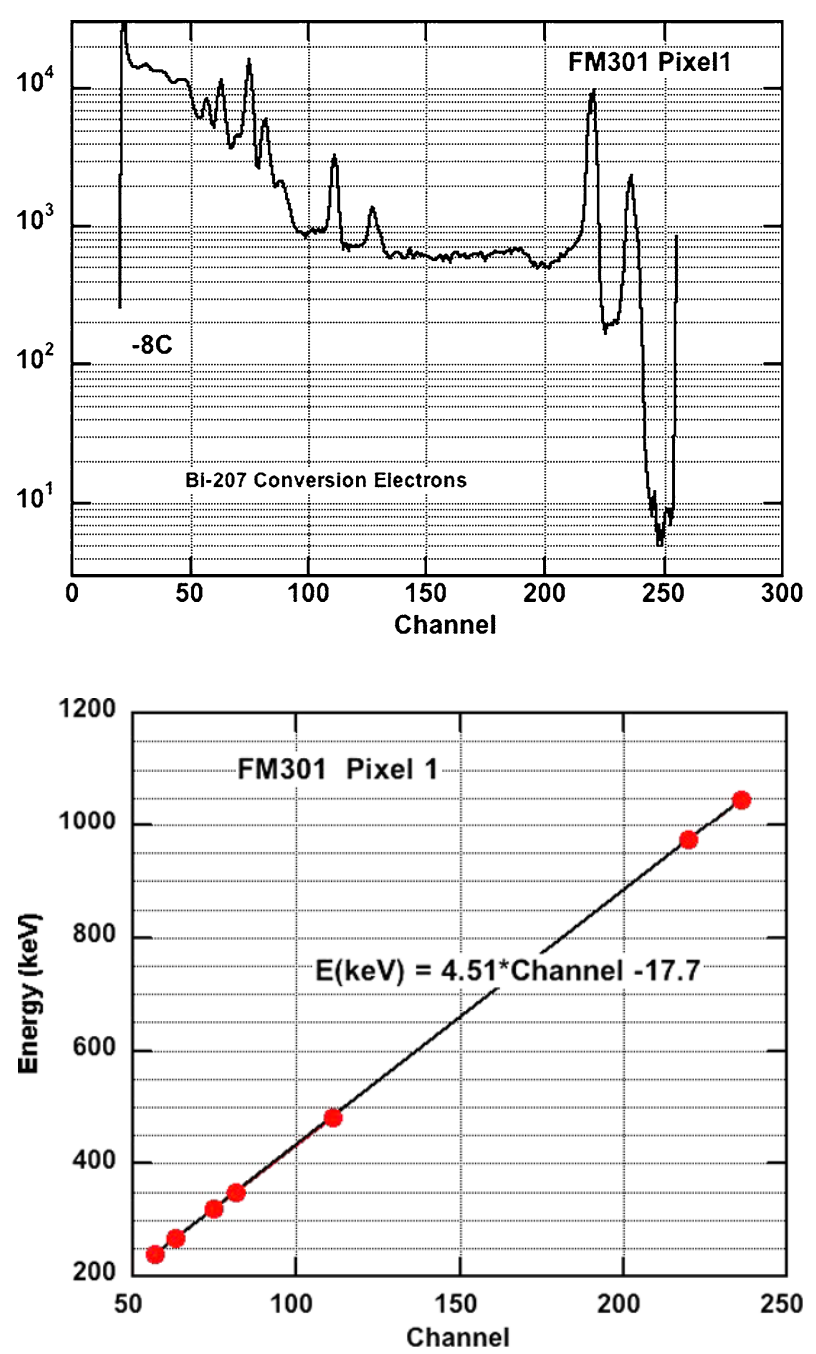

However the pixel-to-pixel variability in offset is sizeable. An average value could not be used in data analysis. The gain/offset measurements summarized here for FM301, a medium energy spectrometer, were carried out for all eight of the flight spectrometers.

\section{MagEIS Digital Processing Unit}

\subsection{Overview}

The Digital Processing Unit (DPU) of the MagEIS instrument is the base interface between the spacecraft and the front-end electronics, cf. Fig. 24. The DPU on a very top level converts the raw data from the Mapper Hybrids and sends environment information back to the spacecraft. This level 0 data is properly formatted, compressed, aligned and tagged so the spacecraft can sort it properly. The DPU also controls ancillary functions to the mission such 
Fig. 22 The temperature dependence of the gains in the nine channels of the FM301 focal plane is summarized

Fig. 23 A summary is plotted of the temperature dependence of the offsets in FM301
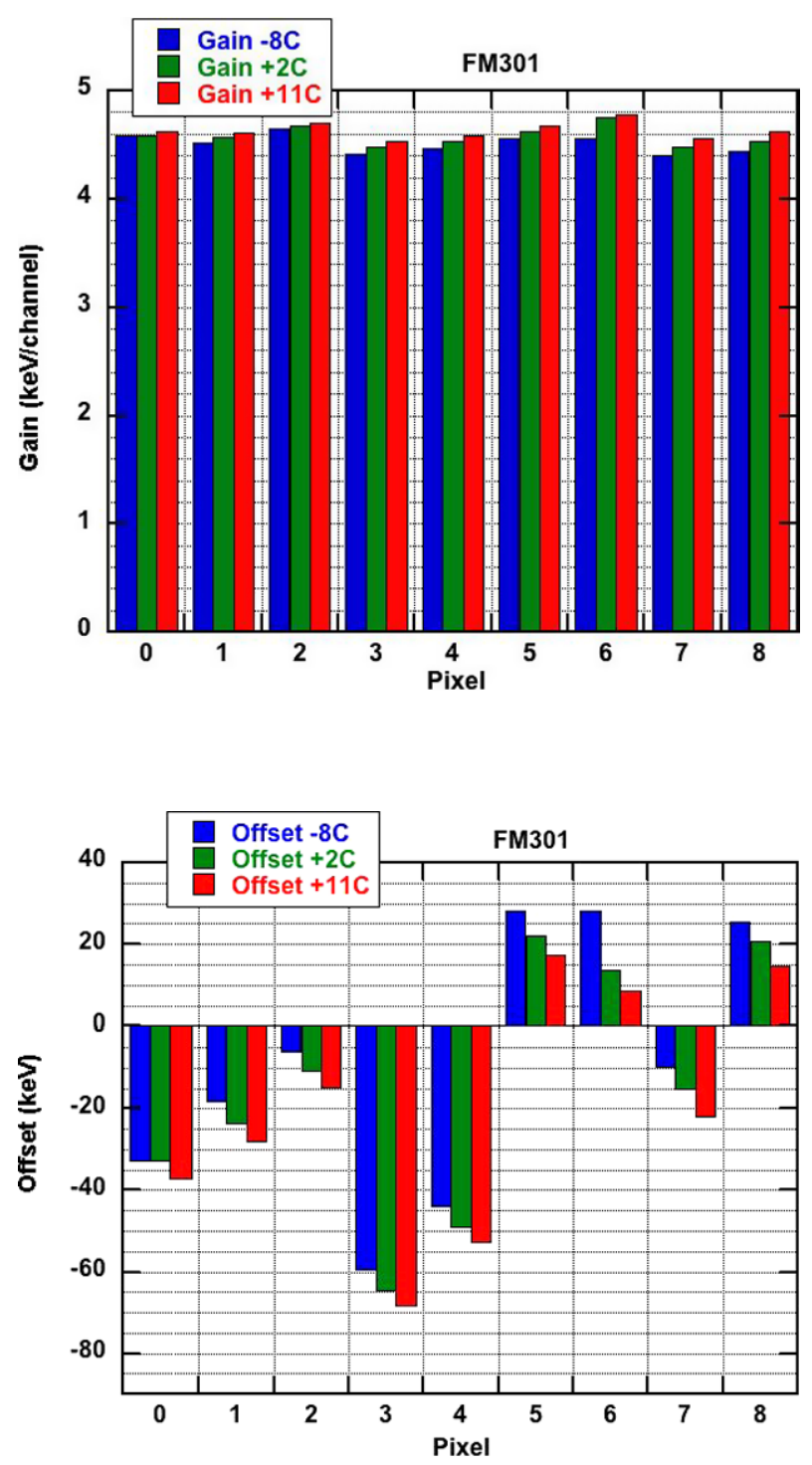

as heaters and MAPPER configurations along with collecting various analog housekeeping data throughout the system.

There are two similar yet quite different DPU designs. The Low/Medium design includes only one electron MAPPER interface. It is less complicated but allows for a higher throughput in electron data. The High design has two MAPPER interfaces, one for protons and the other for electrons. In addition, the electrons are processed through a separate coincidence logic function to allow only those events that meet certain coincidence requirements. This lowers the throughput by eliminating data upfront, but improves background reduction in the science data. 


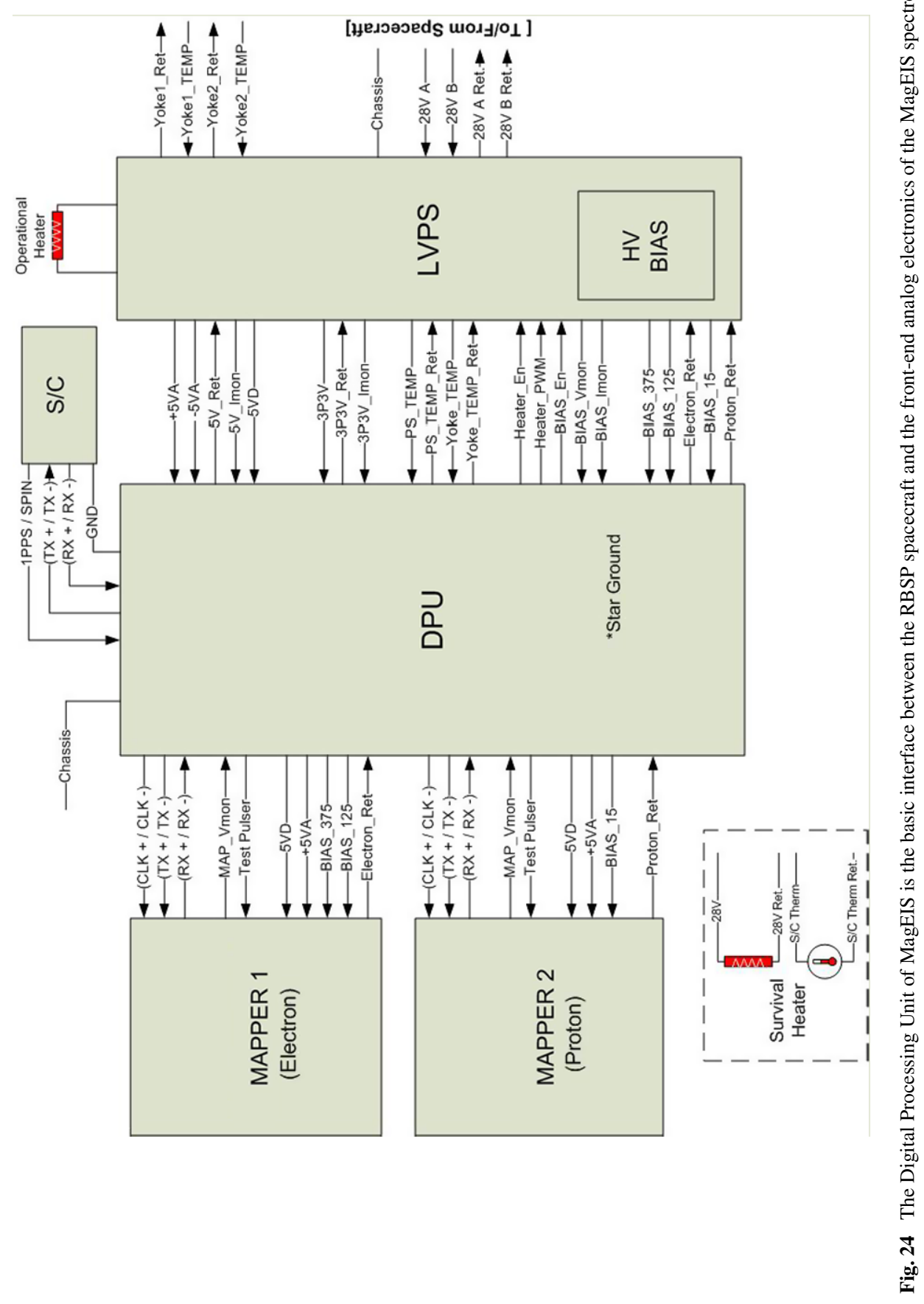




\subsection{DPU Design}

In essence, the DPU is simply a highly advanced and specific CPU (central processing unit) implemented inside of a radiation hardened FPGA. The main components of the FPGA are the CPU core and the Event Logic. The CPU is a modified 8051 core that has the ability to control all functions of the instrument. This achieved by interfacing to registers and external I/Os. The CPU is connected to an RS232 interface running over LVDS to the spacecraft, allowing real time communication and configuration of the system. The Event logic is responsible for interfacing to the MAPPER ICs, data collection, sorting, summing and storage. Both of these components must share the use of memory spaces. Figure 25 shows a breakdown of the FPGA and DPU functions via a block diagram.

\subsection{Software}

The CPU runs a custom operating system. This software, by controlling the CPU, allows near unlimited configurability to the entire system. Commands can be sent from the ground to tune and tweak the settings of the DPU to make simple output parameter and threshold changes. Alternatively, new software can be uploaded at any time to drastically reconfigure the instrument to change performance, alter telemetry priorities, or even change packet formatting. In addition Lookup Tables (LUTs) can be uploaded at any time to alter the categorization of the actual event data.

\subsubsection{Configurable Parameters}

Lookup Tables The lookup tables are the heart of the data "sorting." These tables are configured in such a manner to narrow down the scope of the data to specific areas of scientific interest. This has a twofold benefit. Primarily, regions of data that are superfluous to the mission, considered unimportant or classified as "noise" can be stripped out right at the beginning of the acquisition process. Secondly, this eliminates unnecessary data transmission and reduces the telemetry requirements for each instrument.

MAPPER Settings The MAPPER hybrid is an analog detector front end coupled with digital readout electronics. These Hybrids are commanded through the DPU/spacecraft interface. Changeable parameters include: enabling or disabling individual channels; setting coarse ranges for channel banks; and setting fine threshold ranges for each channel.

Housekeeping Data In addition to flight data, the DPU also collects telemetry from the entire instrument system. This information is gathered once per second and sent to the S/C. This telemetry includes: temperatures throughout the instrument; operating voltages and currents; detector bias voltage settings; calibration test pulser level.

Digital Status The current instrument configuration needs to be known at all times. Once per second, this status information is sent to the S/C. It includes: instrument identification information; software version; spin \& pulse per second monitoring; hybrid command monitoring; packet errors; instrument operational modes; and bias, heater and test pulser status.

Auxiliary Controls The DPU also controls other functions that are critical to the mission. The detector BIAS can be turned on or off. The Operational Heaters can be enabled or disabled. Furthermore, the heater set point can be adjusted to allow fine control over the temperature range for the yoke and associated electronics. 


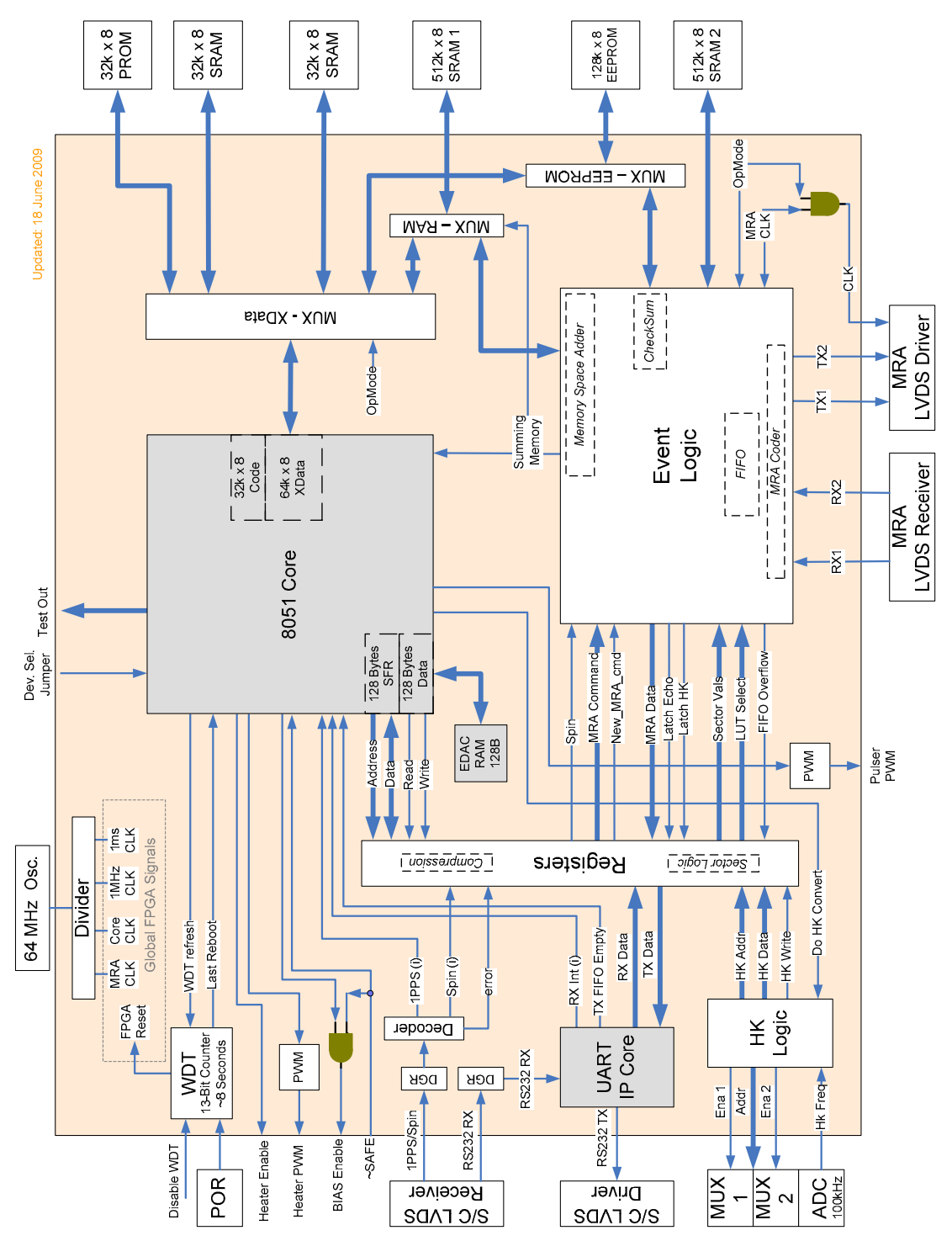

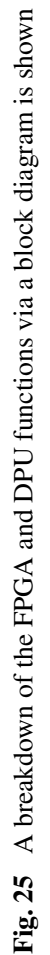


DPU Memory Management The challenge in designing the DPU system was the storage of data. Only one main component (either the CPU or the Event Logic) can access memory at any given time. Whenever the CPU accesses memory, dead time is present for the instrument as no new events can be stored. The standard operating procedure in such situations is to have two memories and ping pong them, or swap them. As one memory is being written to, the other could be read out eliminating any dead time. This was not possible in our system as different data integration times were programmable. For example, histogram data could acquired for minutes at a time, while singles rates were read out every spin. Ping ponging memories in this instance would be impossible.

The solution was achieved by marrying one memory to the CPU for processing and transmission, and one memory to the Event Logic for data acquisition. Then, once every spin (12 seconds nominal), the front end would be ignored while the data was "summed." This process would read the data from both memories, sum them, and then write the new total to the memory controlled by the CPU. Simultaneously, the Event Logic data would be cleared to allow the acquisition of new event counts. This process, which occurs at the very beginning of a spin, takes $13 \mathrm{~ms}$ in the Low/Medium units and $6 \mathrm{~ms}$ in the High unit, which leads to an acceptable dead time of no more than approximately $1 / 10$ of $1 \%$.

DPU Data Flow Each time an electron strikes a detector, a single event is generated inside the MAPPER. This event is processed in an event data flow as described below.

Event Reading The events are transmitted to the DPU each time they are received. It is the responsibility of the DPU to be able to handle simultaneous event readouts from all 10detector channels for a combined possible maximum of 1 million samples per second. The DPU categorizes each event by its source pixel and places the event into a FIFO. The FIFO buffer allows the writing and reading operations to be independent, divorcing the timing of the two and making the system more flexible.

Lookup Tables When the FIFO is not empty, the events are read out and processed. Based on the type of event and the source, each event has the potential to create multiple data entries. Figure 26 illustrates the process in a HIGH unit. The events are then processed through a lookup table. Each lookup table is a $4 \mathrm{~K}$ entry into the EEPROM. This table takes in an event pixel number and a PHA value to determine in which "channel" to place the entry. Each memory type has a different number of allowed channels and is stored in separate locations in memory.

Memory Storage Each spin is broken up into sectors so that it can be determined from which spatial direction the event originated. This sector size is independently configurable for each type of data. Once a channel has been determined from the Lookup Table, the event is then combined with the current sector information and written to memory. The storage occurs in the form of a single count. The memory location for that sector/channel combination is read out, incremented, and then written back to the same location. Each location has the ability to store 24 bits of counts, or in excess of 16 million events. The benefit of counters this large is to allow lengthy integration times on the S/C, lowering the size of transmitted telemetry, increasing the scientific value of individual packets, and reducing the effort needed to analyze and consolidate data on the ground.

High Unit-Electrons The High unit, as briefly described before, has significantly different Event Logic from the Low/Medium unit. The first primary difference is the inclusion 


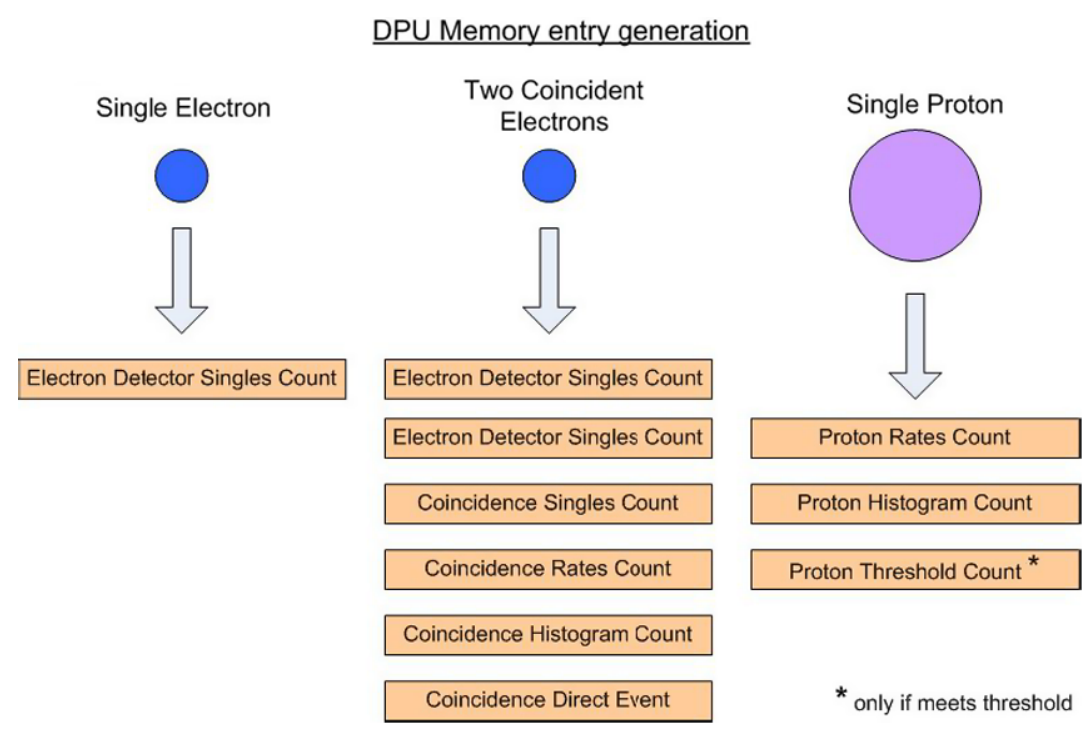

Fig. 26 A block diagram of event generation is shown for the High unit

of a coincidence logic block. This logic determines whether two separate detector events are in coincidence with each other, and therefore the same particle. This is determined by a number of ground programmable parameters such as: time between events; order of events; and channels affected.

Once a particle has been determined to be in time coincidence in the detector stack, processing is enabled and the event is stored in memory. The event is then sorted into a number of categories. A new category in the High unit is the Direct Event Data. This category has the ability to store the actual event PHA data in memory for the last 8 coincidences in a telemetry sample, cf. Fig. 27.

High Unit-Protons With an additional proton MAPPER, a separate Event Logic block is needed. This logic block is similar to the Low/Medium electron processing. However, there are only three detectors and hence only 3 channels available to process. In addition, programmable threshold counters are included to count only events that meet a minimum PHA threshold value. See Fig. 28 for a block diagram of the Proton event processing.

\section{Magnetic Spectrometers Data Outputs}

The magnetic spectrometers have several types of data output as discussed below.

\subsection{Primary and Derived Data-Magnetic Electrons Spectrometers}

The primary data are telemetered 20 times per s/c rotation, or nominally 20 times per 12 seconds. These data are from the 9 pixels in the spectrometer focal plane. Figure 29 shows calibration data from one of the flight medium spectrometers taken using a ${ }^{90} \mathrm{Sr}-{ }^{90} \mathrm{Y}$ beta source, with data acquired from only 4 pixels shown for clarity.

The primary data are counts within the energy pass band delineated by the colored pillars in the plot. The pass band is selected to encompass those energies in the center of the 


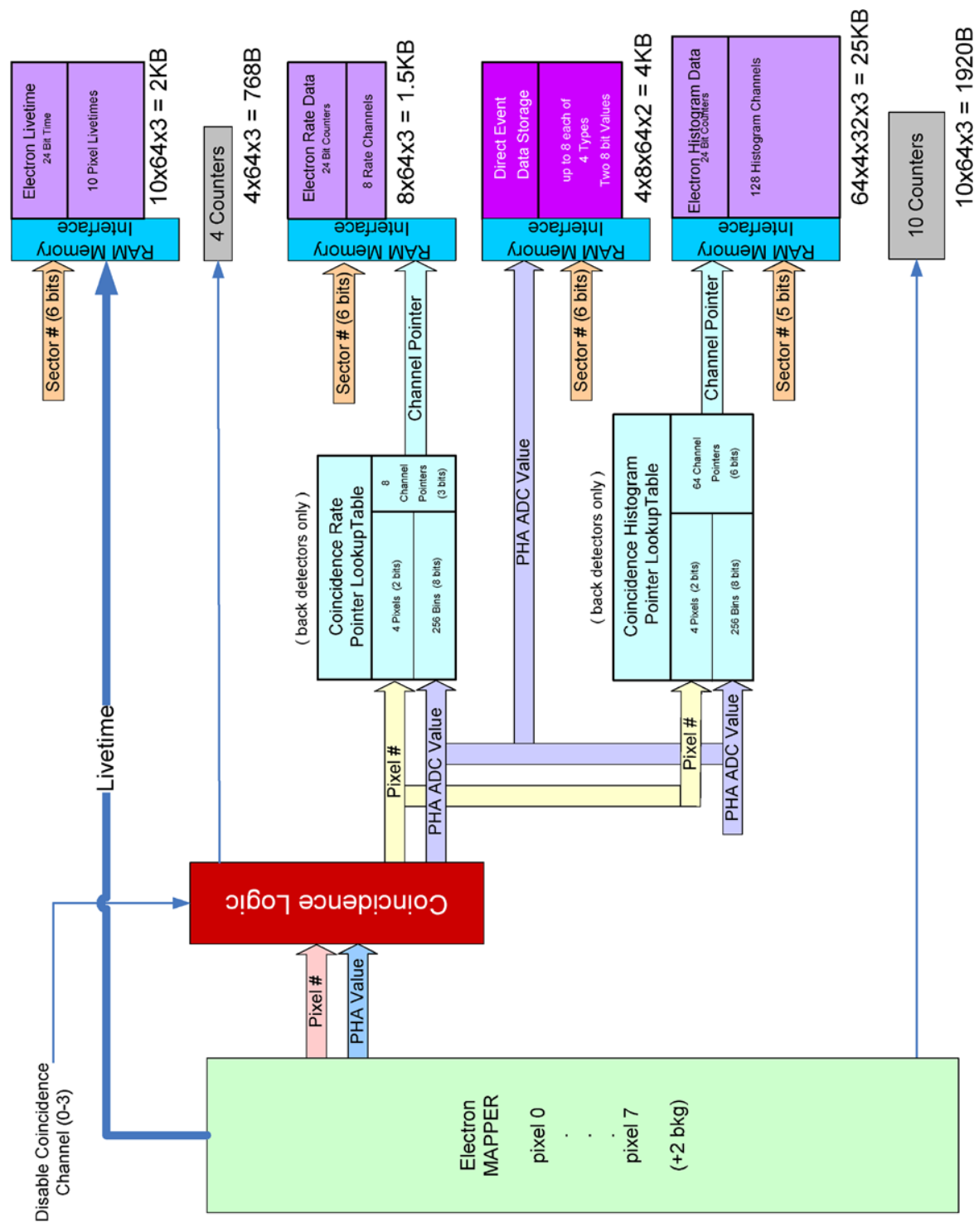

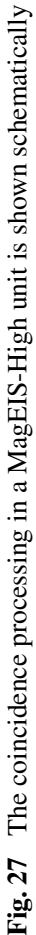




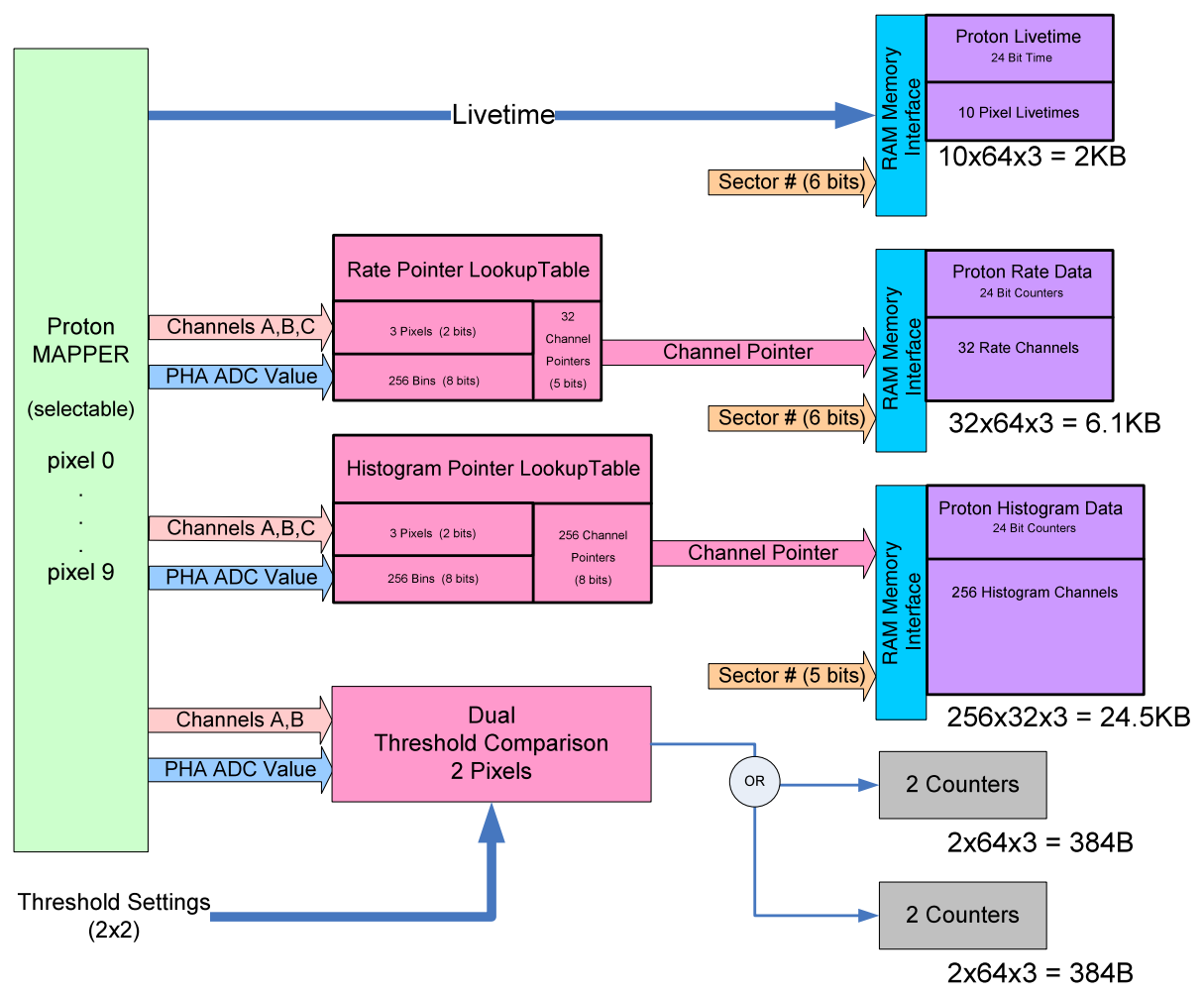

Fig. 28 The proton event processing is shown schematically

Fig. 29 An example of calibration data acquired with a Medium unit is shown

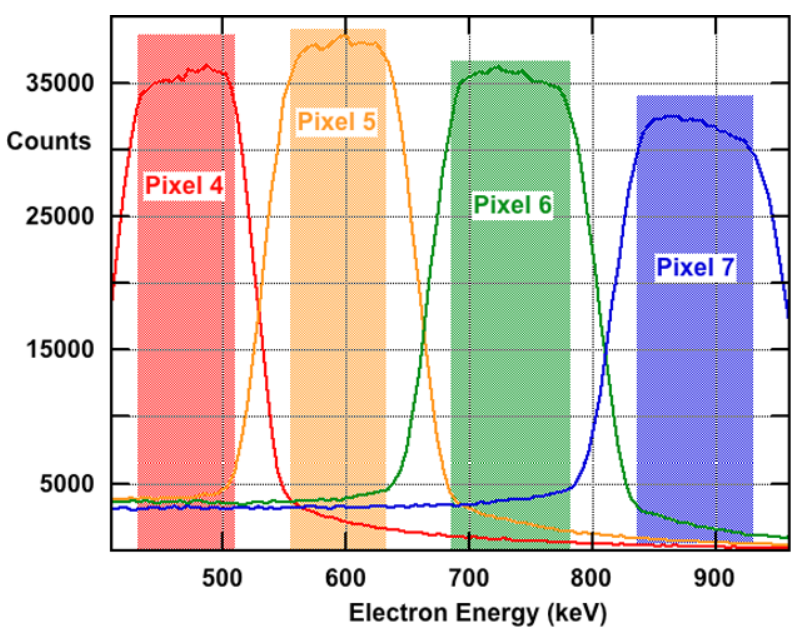

pass band where the pixel response is quite independent of energy, making conversion from counts to differential flux straightforward.

The derived data are telemetered at the same rate and are derived by combining data from adjacent pixels. Thus all counts, whether in the peak or the skirts of a pixel pass band are 
Fig. 30 The figure illustrates schematically how histograms are selected for a given pixel

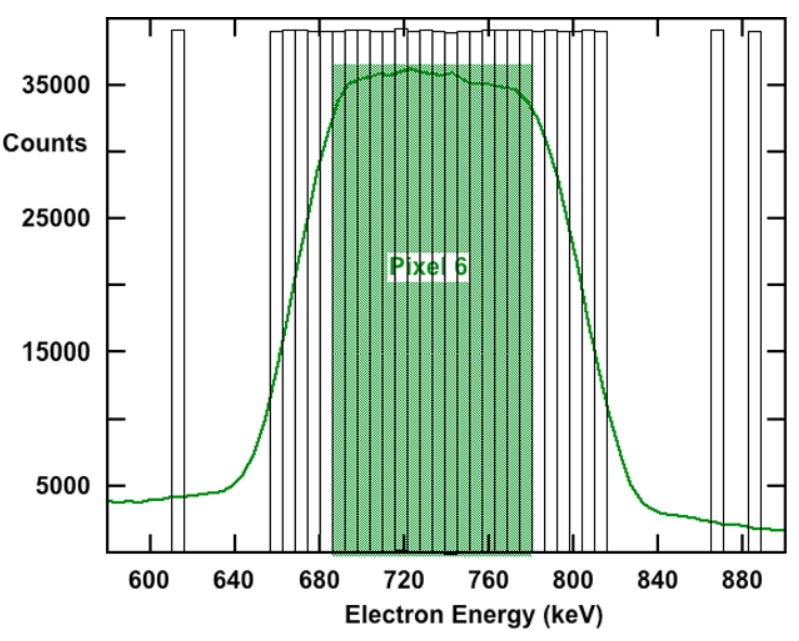

retained and telemetered. The pixel pass bands stored onboard are based upon calibration data, and may be modified during the mission by ground command.

\subsection{Histogram Data-Magnetic Electron Spectrometers}

Every event in every pixel is pulse height analyzed into 256 channels between comfortably above the noise to near saturation. In principle all these data could be telemetered. However it would require far greater telemetry resources than are available. So this high-resolution data are combined to provide the primary and derived data described above.

However some histogram data are saved and telemetered at a lower rate than the primary and derived data. To reduce the telemetry burden, only 64 of the 256 histogram bins are saved. Most of these energy bins cover the region in energy space occupied by a given pixel as shown notionally in Fig. 30 for Pixel 6. However some outlier bins are also saved to monitor background in that pixel. These histogram data are collected for the same 20 sectors as the primary data but integrated over 8 rotations of the RBSP spacecraft.

One of the critical reasons to save histogram data is to use it to determine the background level and to use it to remove background from the science data. Data taken during ground testing of a medium spectrometer is shown in Fig. 31. The same data used in Fig. 29 is used in this figure to plot only the Pixel 4 response in red. The curve in blue is the response outside of the Pixel 4 pass band and the dashed red curve the Pixel 4 response with the background subtracted. In this exemplary case the background subtraction is not really needed because of the strength of the true signal. However one can see that were the signal only modestly above the background and especially if the true signal varied over the pixel pass band, the subtraction process would enable extraction of the true signal from a large background.

The silicon detectors have much better energy resolution than the width of the energy channel in momentum space. This pixel width is determined by size of each pixel along the focal plane. In principal many more, smaller pixels could have been employed, but required spacecraft resources, especially telemetry would be substantially increased. The present selection comfortably meets the mission science requirement. 
Fig. 31 Calibration data acquired with a flight Medium instrument is plotted, clearly showing the pass band and the high and low energy skirts of the pixel response

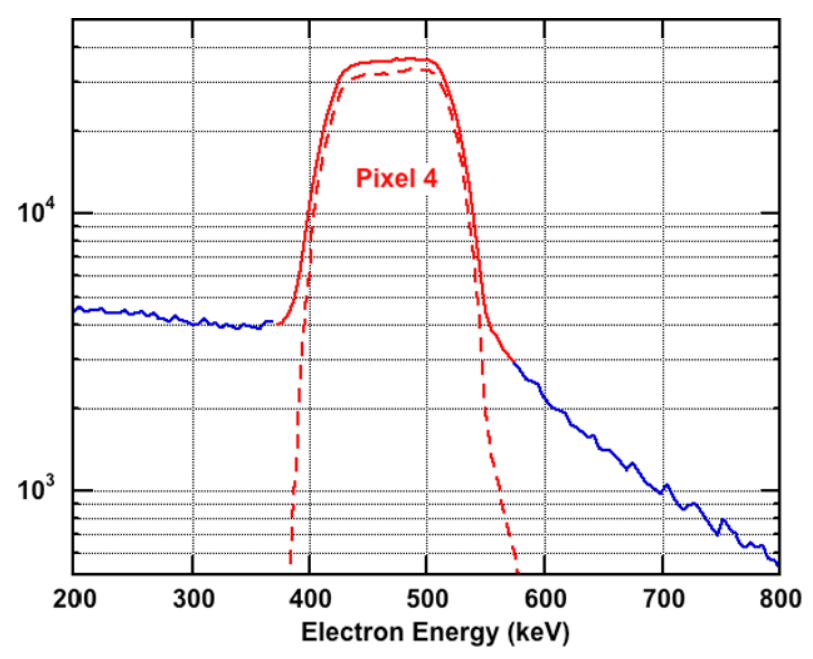

Table 4 Proton Telescope data types, number of channels per type, angular sectors per type, spins/set for a data type and resulting TM rates

\begin{tabular}{|c|c|c|c|c|c|}
\hline $\begin{array}{l}\text { Proton } \\
\text { Telescopes }\end{array}$ & $\begin{array}{l}\text { No. of } \\
\text { Counters }\end{array}$ & Bits & $\begin{array}{l}\text { No. }^{\dagger} \\
\text { Sects. }\end{array}$ & bps $^{*}$ & $\begin{array}{l}\text { Spins }^{\ddagger} \\
\text { per Set }\end{array}$ \\
\hline Main Rates & 32 & 10 & 24 & 326 & 2 \\
\hline Histogram & 256 & 10 & 24 & 544 & 10 \\
\hline Threshold Rates & 4 & 10 & 24 & 25 & 4 \\
\hline Livetime & 10 & 10 & 24 & 219 & 1 \\
\hline
\end{tabular}

\subsection{Proton Telescope Data Outputs}

The process of mapping the telescope detector outputs to data channels is summarized in Fig. 32. In panel [a], the label LP (low proton) is the $10 \mu$ detector in Telescope B while the ORTEC detectors are in Telescope A (see above). The MPA detector is the front detector in both telescopes. The three detectors in each telescope are designated as A, B, and C in panel [a] of Fig. 32 along with their DPU/FPGA identification code $(00,01,10)$. These correspond to the $\mathrm{A}, \mathrm{B}$, and $\mathrm{C}$ designations in panel [b].

Figure 32 panel [b] shows the relationships between the detector/MAPPER outputs (left), the lookup tables (center) and the data outputs (right). 10-bit compressed values, corresponding to the Live times for each of the detector-amplifier-PHA chains, are output to the data stream for each angular sector. The A, B, and C lookup tables are used to define the contents of the Main Rate data (up to 32 channels) and the Histogram data outputs (256 values). The Histogram can be composed of PHA data channels from any combination of the three detectors. Above, we described taking the outputs from A and B detectors to form two 128channel histograms for each.

The detector outputs labeled A and B in Fig. 32 are used to generate four output data channels based on commandable threshold values, two for each detector as described earlier. These are in addition to the Histogram and Main Rate data.

Typical data output from the telescope is summarized in Table 4 where a satellite spin period of $\sim 12 \mathrm{sec}$. was assumed. 
[a]
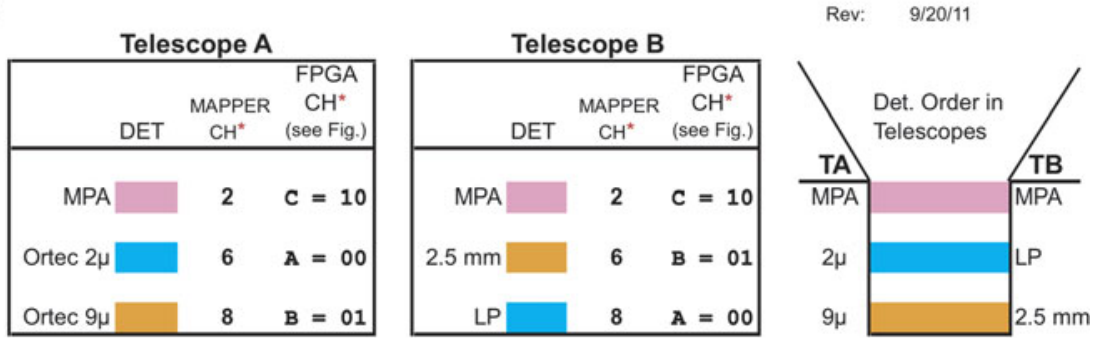

* Commands are used to link the MAPPER CHs to FPGA CHs

[b]

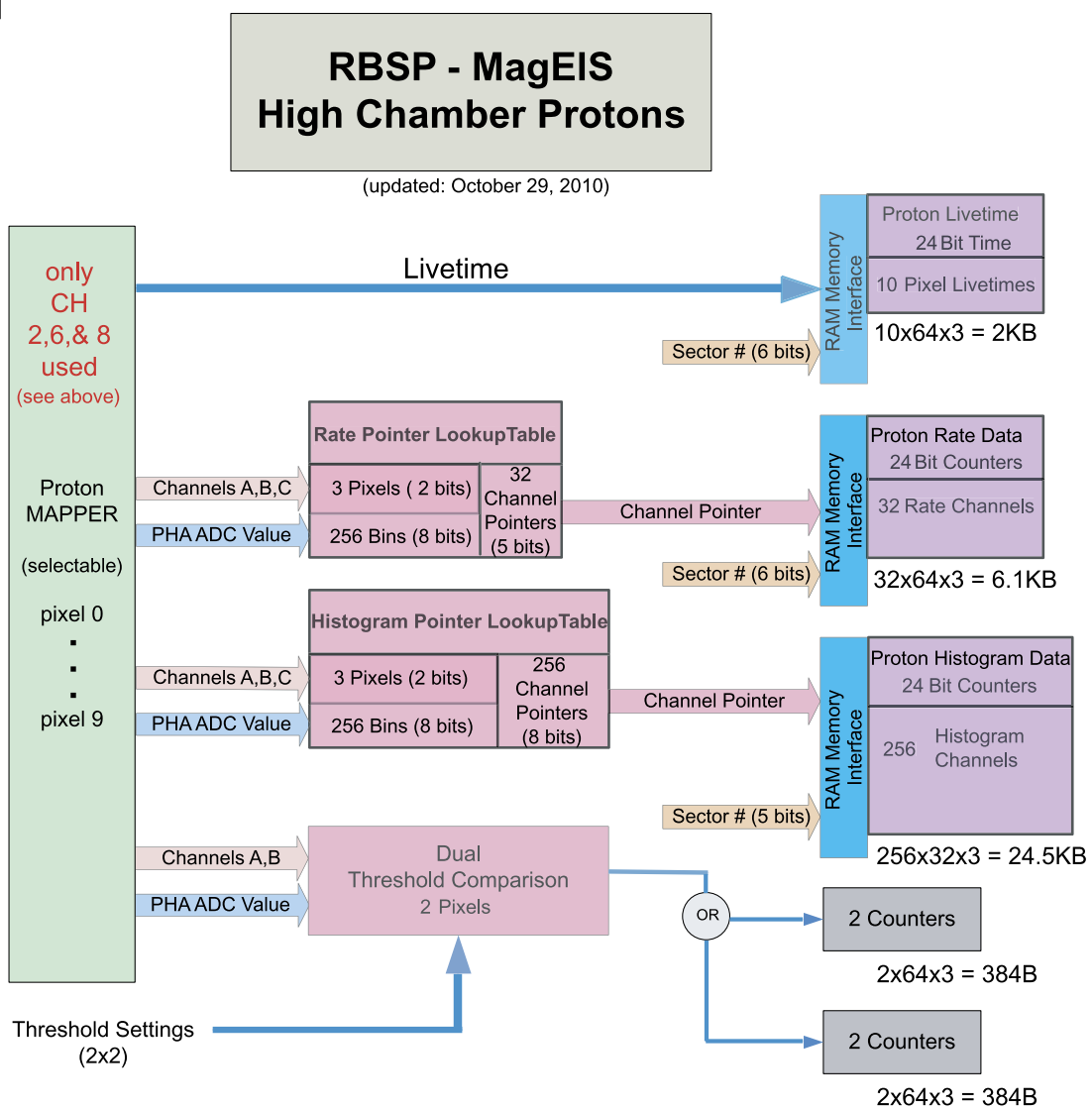

Fig. 32 (a) Block diagram of the proton telescopes, A and B identifying the mapper amplifier channels used and the DPU's FPGA code for the channels; (b) Block diagram showing the mapping of the telescope detector outputs through the DPU/FPGA logic to the output data counters

In this example, the satellite spin is broken into 24 equal sized angular sectors. The data counters are 24 bits in depth and are compressed to 10 bits (see discussion of compression counters). In this example the data, except for the live times, are accumulated over multiple satellite spins (spins per set) before transmission. The telescope data rates in bits per second are show for each type of data. The number of sectors and spins/set are used to optimize 
Fig. 33 The energy range of the electrons from Pm-147 covers the entire range of the low unit, with the intensity in the last pixel, $\mathrm{P} 8$, much less than the others because of the beta spectrum endpoint of $224 \mathrm{keV}$. The ordinate is counts per bin over the test and is arbitrary

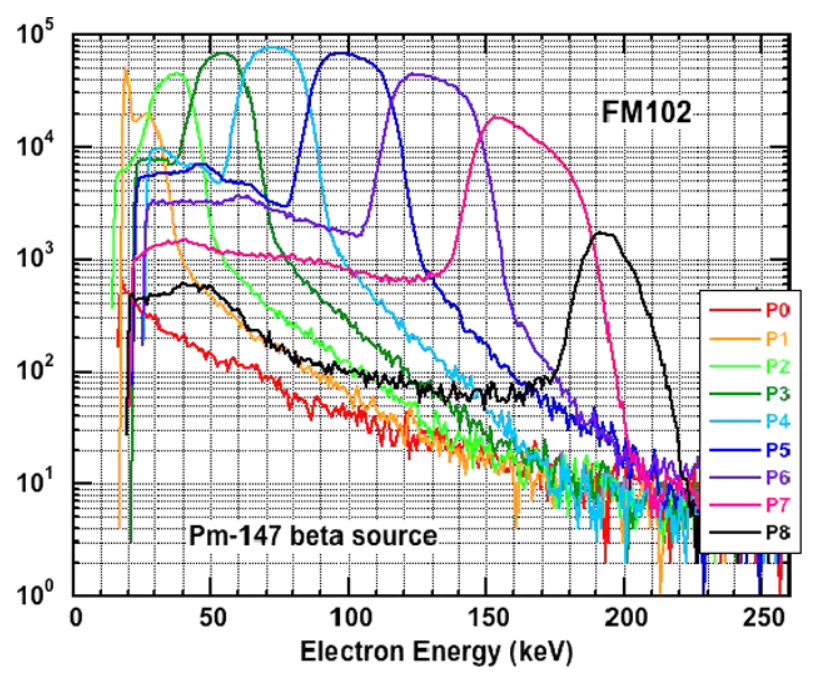

the data sampling while constraining the telemetry rate required. For example, one could measure the Main and Threshold rates plus Live times every spin by reducing the number of angular sectors for all types and increasing the number of spins per Histogram data set.

\section{Electron Spectrometer Performance Verification}

A few representative examples of the performance of the magnetic electron spectrometers are shown, beginning with a low unit. A low-energy spectrometer was placed in a vacuum chamber and a Pm-147 beta emitter was placed a few inches in front of the spectrometer aperture, Fig. 33 shows the FM102 unit (Flight Model 102) response. The endpoint of the beta spectrum from Pm-147 is at $224 \mathrm{keV}$. Data were collected from all pixels. Figure 34 is an expanded plot showing the response of the FM102 lowest four energy channels to the Pm147 source (see caption). It can be seen at a glance that the response of each pixel is within its respective pass band as designed. As noted elsewhere in this paper, pulse-height analysis removes out-of-band responses, and the histogram data permit removal of the background within the pass band if deemed necessary.

The same test described above was carried out using a Ni-63 source in place of Pm-147, Fig. 35. The endpoint energy of the Ni-63 beta spectrum is $67 \mathrm{keV}$. Figure 35 shows that the threshold of P0 is below the $20 \mathrm{keV}$ noise threshold and measures only background. It will be used in flight to monitor the penetrating background from energetic protons, and from bremsstrahlung.

The next series of plots show the response of a medium-energy spectrometer. Figure 36 shows the response of FM201 to a Sr-90/Y-90 beta source. The beta spectra from this mother-daughter pair have endpoint energies of $546 \mathrm{keV}$ and $2280 \mathrm{keV}$ respectively. The energy range of the electrons from Sr-90/Y-90 covers much more than the entire range of a medium spectrometer. As a result the count rate in most of the pixels of FM201 are much the same. The modest difference seen in P4 (pixel 4) from its neighbors, P3 and P5, is due to the endpoint of the Sr-90 spectrum at $546 \mathrm{keV}$, that is, the energy resolution of the spectrometer is such that details in the electron energy spectra emitted by the test source can be seen.

The response of a medium-energy spectrometer can be seen more clearly by using a beta source with a lower endpoint energy, Pm-147. To help illustrate the fact that the responses 
Fig. 34 Data acquired during a Pm-147 test are displayed at lower energies only in order to show the response in pixels 1 through 4 more clearly. The black arrow shows the threshold of P1, which can be seen to be just below $20 \mathrm{keV}$. The ordinate is counts per bin over the test and is arbitrary. Small differences between Fig. 33 and Fig. 34 are a result of small changes in laboratory temperature. The ordinate is counts per bin over the test and is arbitrary
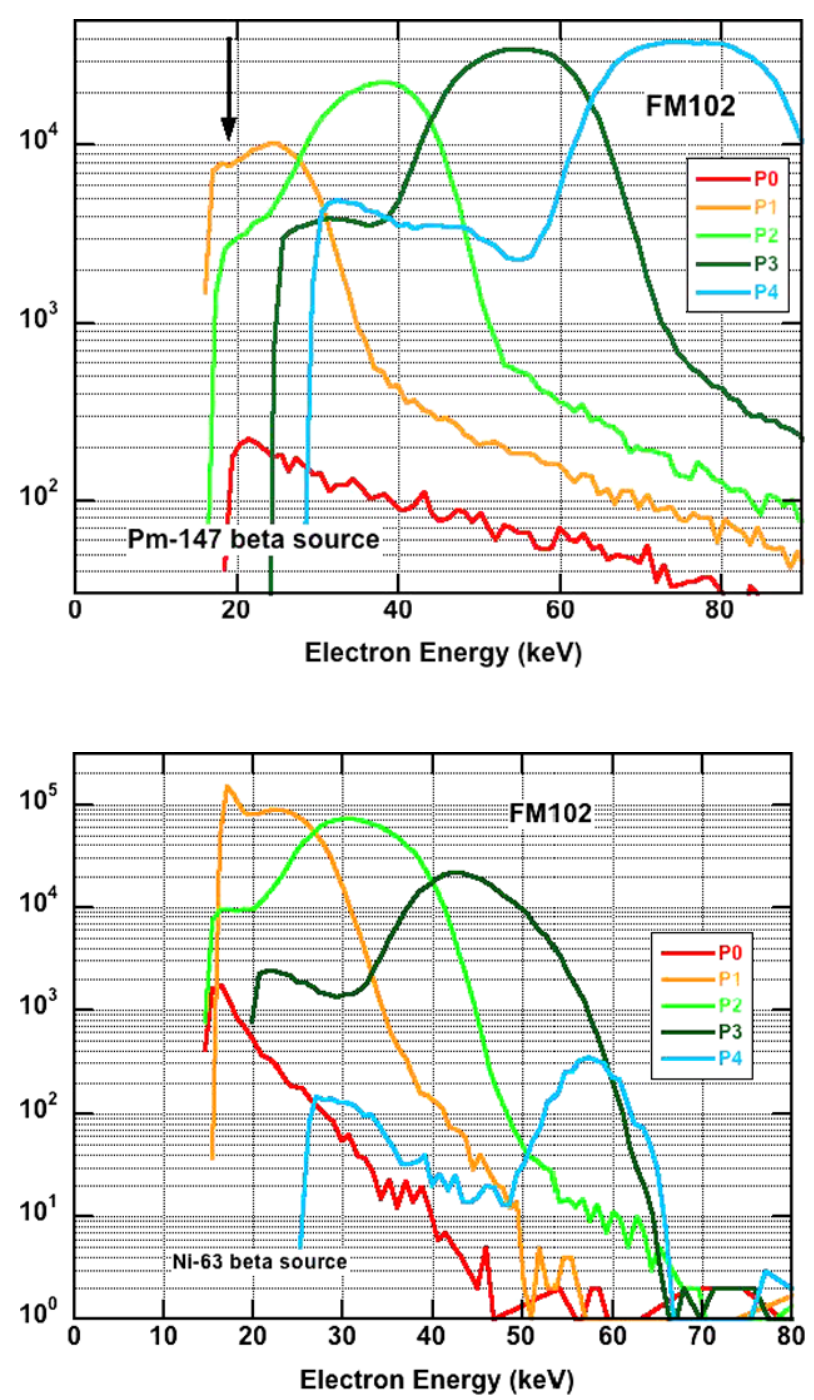

Fig. 35 The response of a low-energy spectrometer to a Ni-63 source is shown. The response in $\mathrm{P} 4$ ends at $\sim 67 \mathrm{keV}$, the endpoint of the Ni-63 beta spectrum. The ordinate is counts per bin over the test and is arbitrary

of the multiple units of the same type are essentially identical, another medium spectrometer was used to acquire the data used in Fig. 37. (FM302-Med35 in RBSP-B rather than FM201-Med75 in RBSP-A).

The Pm-147 beta spectrum nicely illuminates $\mathrm{P} 0$, showing a pass band of $\sim 75-110 \mathrm{keV}$. The rapidly falling beta energy spectrum causes the response of P1 to fall substantially over its energy range, and the response in $\mathrm{P} 2$ is very small for the same reason. Note the absence of counts in P2 above the Pm-147 endpoint energy of $224 \mathrm{keV}$. The three higher-energy channels that are plotted show a small background response in channels below their pass bands, counts which would be removed by pulse-height analysis during data analysis if this were flight data.

For this run the source was placed just outside of the aperture of the spectrometer in order to get a high count rate and some pileup. The regions of pileup in $\mathrm{P} 0$ and $\mathrm{P} 1$ are labeled. The 
Fig. 36 The response of a medium-energy spectrometer to a Sr-90-Y-90 beta source is shown. The ordinate is counts per bin over the test and is arbitrary
Fig. 37 The response of a medium-energy spectrometer to a Pm-147 beta source is shown. The ordinate is counts per bin over the test and is arbitrary
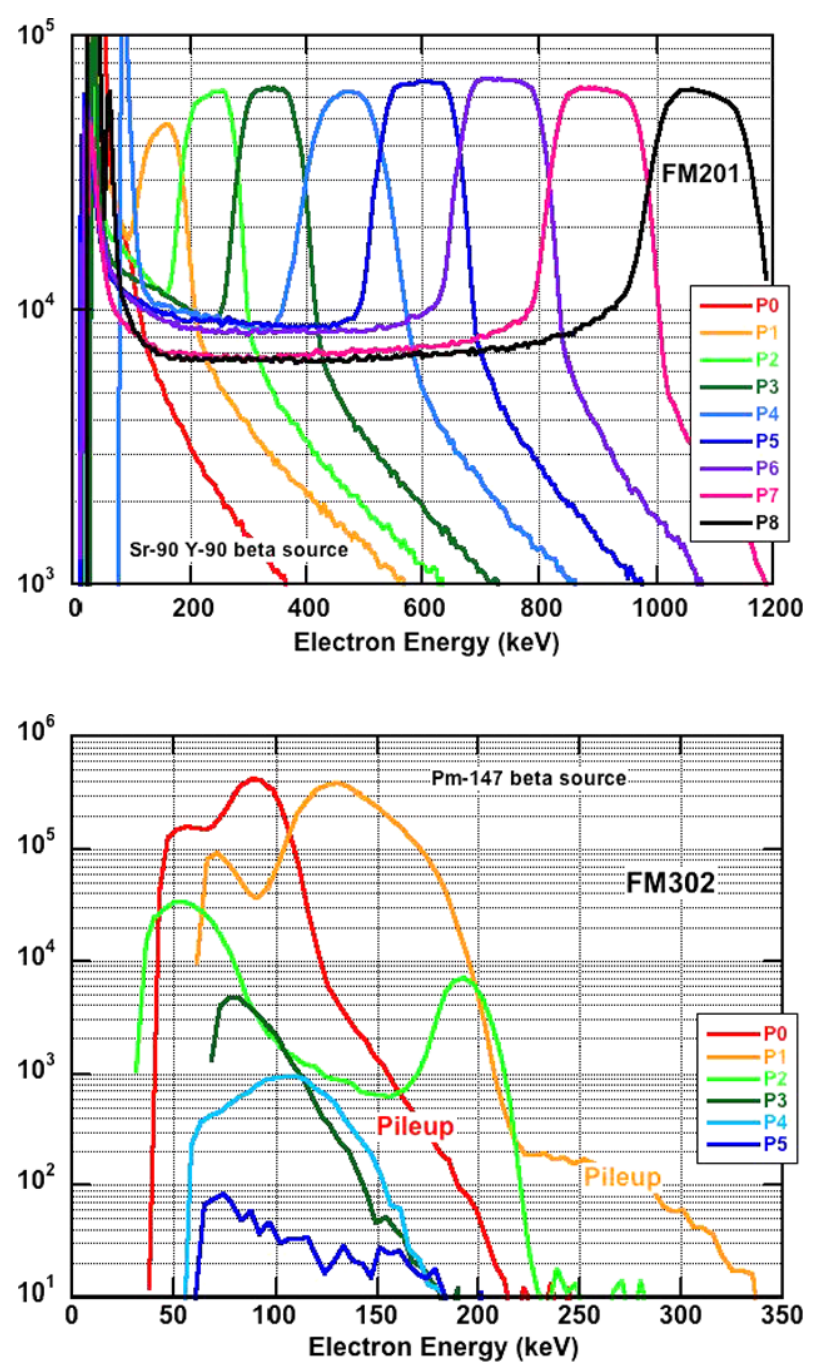

pileup is orders of magnitude smaller that the signal within the pass band, and what there is would be removed by pulse-height analysis if this were flight data.

The response of high-energy spectrometers was measured in a very similar way. A Ru106-Rh-106 beta emitter was placed several inches in front of the spectrometer aperture, Fig. 38. The beta spectra from this mother-daughter pair have endpoint energies of $39 \mathrm{keV}$ and $3541 \mathrm{keV}$ respectively. The Rh-106 decay emits many energetic gamma rays that complicate the observed energy spectra in the high-energy spectrometer. This spectrometer has only four pixels rather than nine but has a two-element stack; there is a P1 front and P1 rear and so on, see discussion in Sect. 3.2. Observations are shown using only the four rear pixels in the figures below.

The response of the instrument nicely characterizes the shape of the Rh-106 beta spectrum, with the endpoint electron energy just above $3500 \mathrm{keV}$ clearly revealed. The source completely illuminates the first three pixels, with just a small contribution to P4, whose endpoint is almost $5 \mathrm{MeV}$. The shoulder in the P4 energy spectrum, labeled Compton edge, 
Fig. 38 The response of a high-energy spectrometer to a $\mathrm{Ru}-106 \mathrm{Rh}-106$ beta source is shown. The ordinate is counts per bin over the test and is arbitrary
Fig. 39 Data acquired from both high-energy spectrometers are plotted on the same scale with normalized amplitudes. The responses of both units to the $\mathrm{Ru}-106 \mathrm{Rh}-106$ beta source are virtually identical. The ordinate is counts per bin over the test and is arbitrary
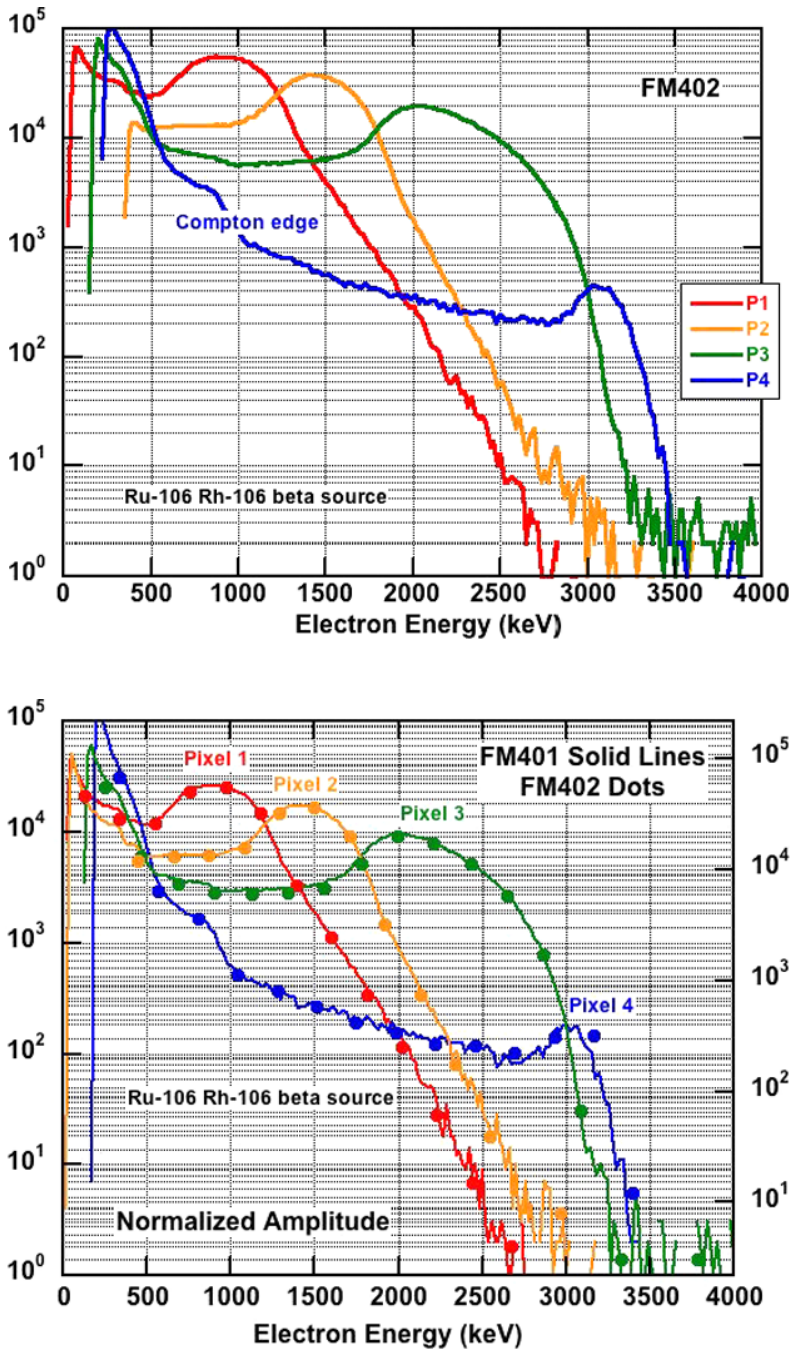

as well as the general level of background in all channels is due to the energetic gammas emitted by the beta source along with the wanted electrons. In flight no such gammas will be present and the background will be substantially less. Nevertheless this test cleanly delineates the pixel pass bands except for P4.

There was a substantial effort expended to make the same instrument types on both spacecraft perform alike. An example of the comparisons that have been made is shown in Fig. 39 where data from the two high-energy spectrometers have been plotted together after being exposed to the Ru-106-Rh-106 source.

\section{MagEIS Data Outputs}

All eight MagEIS units utilize a packet-based organization scheme for the raw instrument telemetry data. Each data type (e.g. count rates, livetime, housekeeping) is organized into a 
binary Consultative Committee for Space Data Systems (CCSDS) telemetry packet, which consists of a header followed by the raw telemetry bytes. The raw telemetry bytes are of variable length depending on the specific data type. Each CCSDS packet is prepended with a ground annotation and a 3-byte generic header by the Mission Operations Center (MOC) at APL. This combination of the generic header, ground annotation and CCSDS telemetry packet is known as a "payload telemetry packet" (PTP). For each of the eight MagEIS units, one daily level 0 binary data file is produced, which consists of all PTP packets for a given data type, for an entire mission day.

Per spacecraft, all four MagEIS units (low, both mediums, and high) are independently operated in either "science" mode or "maintenance" mode. Science mode data consists primarily of the main and derived channel count rates, the histogram data, the livetime data, and the space weather beacon data. Maintenance mode data consists primarily of the housekeeping data, the digital status data, and command echo data, all of which are also available in science mode. When in science mode, the low and both medium units can be commanded into "sample" mode (e.g. burst-rate mode or high-rate mode). Sample mode uses a larger number of sectors per spin than normal science mode, which increases the temporal resolution of the measurements. To accommodate this increased resolution while meeting the nominal telemetry requirements, the derived channel and histogram data are not recorded in sample mode. It is anticipated that sample mode will be used when the spacecraft traverses the midnight-to-noon region of the equatorial magnetosphere, where electron microbursts are most likely to be observed. The high unit does not utilize a sample mode.

The Science Operations Center (SOC) at the Los Alamos National Laboratory (LANL) receives the binary level 0 MagEIS data, organized into daily (mission day) PTP files for each data type. These files are processed into level 1 data at the SOC, using MagEISspecific data processing software written in the Interactive Data Language (IDL, version 8.0) a higher-level data analysis and visualization language. The data processing software unpacks the raw level 0 bytes into time-tagged (by mission elapsed time-MET), sorted data products, converted into physical units. The processed data are saved into International Solar-Terrestrial Physics (ISTP) compliant Common Data Format (CDF, version 3.3.1) level 1 files, organized by UTC day. Once level 1 data has been validated by the MagEIS team, the SOC processes the level 1 data into level 2 data, which involves the conversion of raw count rates into calibrated fluxes. The process also involves applying livetime and background corrections, if deemed necessary, to obtain corrected count rates that are then turned into differential unidirectional fluxes.

For a given UTC day, the level 2 data is contained in eight ISTP-Compliant CDF files, one for each of the four MagEIS units on the two RBSP spacecraft. The following naming convention is used for the data files: rbspx_***_ect-mageiszzz-L2_yyyymmdd_vx.y.z.cdf. Here, $x=\{a, b\}$ is the descriptor for the particular spacecraft and $x x x=\{L O W, M 35, M 75\}$ is the descriptor for the particular MagEIS unit. The yyyymmdd descriptor indicates the UTC day. The vx.y.z descriptor indicates the data version number. Increments in $\mathrm{x}$ are used for major structural changes to the data file that would require a new version of the data processing software. Increments in y are used if the file contents have been updated to be of better quality (e.g. a calibration change). Increments in $\mathrm{z}$ are reserved for minor bug fixes in either the data file or the processing software. In addition to the eight per-unit level 2 files, two additional level 2 data files are produced that combine the spin-averaged electron flux products at a selected number of energy channels across all four MagEIS units on a spacecraft. In all, there are a total of ten level 2 CDF data files from the ECT-MagEIS instruments per day. The naming convention for the two combined data files, providing a continuous electrons energy spectrum over the roughly $30 \mathrm{keV}-4 \mathrm{MeV}$ range, is identical 
Table 5 MagEIS data outputs and modes

Resolution Mode

\begin{tabular}{|c|c|c|c|c|c|c|}
\hline $\begin{array}{l}\text { Low \& Medium ( } 3 \\
\text { units) }\end{array}$ & $\begin{array}{l}\text { Num } \\
\text { PHA } \\
\text { chan/ } \\
\text { pixel }\end{array}$ & $\begin{array}{l}\text { Num data } \\
\text { chan/pixel* }^{*}\end{array}$ & Bits & $\begin{array}{c}\text { Num } \\
\text { sectors/spin }\end{array}$ & bps** & $\begin{array}{c}\text { Spins } \\
\text { /set }\end{array}$ \\
\hline Main Rate & & 9 & 10 & 20 & 172 & 1 \\
\hline Derived Rate & & 7 & 10 & 20 & 132 & 1 \\
\hline Histogram & 64 & 9 & 10 & 20 & 1234 & 8 \\
\hline Livetime & & 10 & 10 & 20 & 184 & 1 \\
\hline
\end{tabular}

\section{High (Electrons)}

\begin{tabular}{|l|c|c|c|c|c|c|} 
Main Rate & & 8 & 10 & 20 & 146 & 1 \\
Detector Singles & & 10 & 10 & 20 & 192 & 1 \\
Coincidence Singles & & 4 & 10 & 20 & 79 & 1 \\
Histogram (rear pixels) & 64 & 4 & 10 & 20 & 567 & 8 \\
Livetime & & 10 & 10 & 20 & 184 & 1 \\
Direct Events & & NA & $\mathbf{5 1 2}$ & 20 & 886 & 1 \\
\hline
\end{tabular}

High (Protons)
\begin{tabular}{|l|c|c|c|c|c|c|} 
Main Rate & 32 & 10 & 24 & 652 & 1 \\
Histogram (3 Pixels) & 256 & 1 & 10 & 24 & 680 & 8 \\
Threshold Rates & & 4 & 10 & 24 & 91 & 1 \\
Livetime & & 4 & 10 & 24 & 91 & 1 \\
\hline
\end{tabular}

\section{Sample Mode}

Low \& Medium Units
(Support High Sector

Rates)

\begin{tabular}{|l|c|c|c|c|c|}
\hline High Rate (2 Units) & 3 & 8 & 1000 & 2036 & 1 \\
Main Rate (3 units) & 9 & 10 & 20 & 172 & 1 \\
Livetime (3 units) & 10 & 10 & 20 & 184 & 1 \\
\cline { 2 - 5 } & & same as in Resolution Mode (electrons) & same as in Resolution Mode \\
High (protons) & \multicolumn{5}{c|}{}
\end{tabular}

* pixel for histogram, channel for all other types

** depends on number of sectors/spin and spins/set values in red are changeable by command

to that given above but with the "zzz" unit descriptor omitted. The ISTP-compliant level 2 MagEIS CDF files are fully compatible with standard data visualization software packages, such as Autoplot (autoplot.org) and NASA CDAWeb (cdaweb.gsfc.nasa.gov).

Table 5 shows the nominal telemetry parameters and data bit-rates for the various MagEIS data products from all four electron detectors and the proton telescope on each spacecraft. The first three sub-tables show the nominal telemetry parameters for normal science mode ("resolution mode"), while the last sub-table shows the parameters for the burst mode or high rate mode ("sample mode"). The first column in the sub-tables denotes the data product in question. The second column gives the number of pulse-height analyzer (PHA) channels, which is only defined for the histogram data products. The third column indicates the number of energy channels for the count rate data products (e.g. main and derived) or the number of detector pixels for the histogram and livetime data. The fourth column denotes the bit depth, where the data compression is either 24 to 10 bit or 16 to 8 bit. The direct event data is uncompressed. The fifth column indicates the number of angular sectors per 
Table 6 Data characteristics

\begin{tabular}{llll}
\hline Instrument & Data Type & Compression & Error \\
\hline Low/Medium & Electron Rates (main \& derived) & 24 to 10 & $3 \%$ \\
Low/Medium & Electron Samples (high rate) & 16 to 8 & $3 \%$ \\
Low/Medium & Electron Livetimes & 24 to 10 & $3 \%$ \\
Low/Medium & Electron Histograms & 24 to 10 & $3 \%$ \\
High & Electron Coincidence Rates & 24 to 10 & $3 \%$ \\
High & Electron Coincidence Histograms & 24 to 10 & $3 \%$ \\
High & Electron Coincidence Singles & 24 to 10 & $0 \%$ \\
High & Electron Coincidence Direct Events & none & $3 \%$ \\
High & Electron Coincidence Detector Singles & 24 to 10 & $3 \%$ \\
High & Electron Rate Livetimes & 24 to 10 & $3 \%$ \\
High & Proton Rates & 24 to 10 & $3 \%$ \\
High & Proton Histograms & 24 to 10 & $3 \%$ \\
High & Proton Singles (Threshold) & 24 to 10 & $3 \%$ \\
High & Proton Livetimes & 24 to 10 & \\
\hline
\end{tabular}

spin, where each spacecraft spin is divided into an equal number of sectors. The last column indicates the number spin integrations in a given data set, i.e. how many spins the data is accumulated over. All values shown in red are changeable by ground command and can be adjusted in concert to increase or decrease the telemetry bit rate (shown in column 6) as needed. The values of the number of sectors per spin and number of spins per set (columns 5 and 7) are adjusted to maximize the science return while staying within allotted telemetry constraints.

\section{MagEIS Instrument Users Guide-Understanding the Data}

This guide documents the operational features of the instrument. Certain settings and conditions can cause unexpected behavioral patterns in the data sets. While the instrument is operating completely within specifications and constraints, the data may not be exactly what is expected. The goal of this section is to provide an understanding of why the data looks the way it does.

\subsection{Data granularity}

In order to conserve telemetry, data are compressed using one of two schemes: 24 to 10 or 16 to 8. Some data are not compressed at all. Refer to Table 6 for data type and implemented compression scheme. The resulting compression error is the maximum error possible for that data type.

\subsection{Spin Pulse/Sectoring}

The spin pulse occurs asynchronously to the MagEIS DPU clocks. In order to align the DPU clock with the spin pulse, the DPU samples the spin pulse every 1/4 ms. All timing and sector sizes are based off of this $1 / 4 \mathrm{~ms}$ boundary. The minimum delay from the occurrence of the spin pulse until the DPU sector reset is therefore $1 / 4 \mathrm{~ms}$. The maximum delay is 
$1.2 \mathrm{~ms}$. Because of this, the sector resolution and sector error of any given event is $+/-$ $1 / 4 \mathrm{~ms}$.

The spin pulse time communicated by the spacecraft is divided by the requested number of sectors to create a sector size. This sector size is accurate to $1 / 4 \mathrm{~ms}$. However, because of rounding, the final sector may end up shorter than the calculated time. As an example: suppose that the previous spin period was 12.03 seconds. The number of sectors desired is 31 , that is $12.03 / 31=388.064 \mathrm{~ms} / \mathrm{sector}$. This is rounded $u p$ to $388.25 \mathrm{~ms}$, as the boundary is $1 / 4 \mathrm{~ms}$. If the present spin period is exactly the same, this leaves the final sector to be $382.5 \mathrm{~ms}$, or $1.5 \%$ smaller than the first 30 sectors. The discrepancy in counts for the final sector would necessarily coincide with a lower livetime for that sector. However; note that the livetime value itself has an accuracy error of $3 \%$, and may mask, or exaggerate the smaller sector size.

\subsection{Livetime}

In order for the Hybrid to latch and read out the Livetime of the instrument, data acquisition must stop for a short period of time. Because data acquisition stopped, the following Livetime that is read out will appear a few clock cycles smaller than expected. This effect depends on the frequency of the Livetime readout, which is based on the size (in ms) of each sector for the main rates.

In addition, just as the data is compressed to conserve telemetry bandwidth, so is the Livetime. The compression scheme used for the Livetime is the same as the data, allowing for an error of up to $3 \%$. These factors mean changes in sector size will cause a stepped decrease in the Livetime readout.

The current maximum for the Livetime as read out of the Hybrid is a 16 bit counter, or a maximum readout value of 65,536. Each count value represents 32 us. Thus, the maximum possible Livetime is 2.097 seconds. If sectors are defined in such a manner that the resulting sector length is longer than 2.097 seconds, the counter will reach the maximum and stop. It will NOT roll over. This will always result in a specific value of 65,536. If ever a value of 65,536 is shown for the Livetime, the sector should be marked as invalid, because it is impossible to know the true livetime, and most probably it was much higher than that value.

Note that this affects the Hybrid data only. Data stored in the DPU is 24 bits per sector. The instrument will regularly send values larger than 16 bits to the GSE when the data is read out, as multiple sums of the livetime data will be made during flight. The above caveat specifically refers to individual sectors exceeding 2.097 second.

\subsection{Summing}

At the beginning of a spin (as soon as the instrument receives a spin pulse) summing occurs. Summing is the process of aggregating all counts, data and Livetimes from the hardware memory into the software memory. This is a mandatory operation and cannot be disabled. The length of the summing is as follows: Low \& Medium Units, $13 \mathrm{~ms}$; High Unit, $6 \mathrm{~ms}$.

During summing, no data of any kind can be stored, resulting in dead time. To account for the dead time, the summing time should be subtracted from the sector time of the first sector(s), depending on the sector length.

For example: suppose that Low Instrument is running is sample mode, with each sector size set to $10 \mathrm{~ms}$. Because of the summing period of $13 \mathrm{~ms}$, the first sector will not contain any events, regardless of the Livetime readout, as the entire sector occurred during summing. For the second sector, $3 \mathrm{~ms}$ will need to be subtracted from the Livetime, as only the final $7 \mathrm{~ms}$ of the second sector were available for taking data. 


\subsection{Direct Events}

For Electron Coincidences on the High unit, Direct Event data are stored. Each sector can retain both the front and back PHA values for up to 8 events. If less than 8 events occur in any given sector, the remaining allocation for that sector will return 0 s. If more than 8 events occur, you will lose Direct Event data, as the instrument has neither the memory nor the telemetry to retain more. The Direct Events that are transmitted are the last eight events to be acquired during that sector. All events prior to the final eight, regardless of the number, are simply discarded.

Acknowledgements This work was supported by the University of New Hampshire under Contract 10068, the Johns Hopkins Applied Physics Laboratory under Contract 967399, and NASA under contract NAS5-014072.

Open Access This article is distributed under the terms of the Creative Commons Attribution License which permits any use, distribution, and reproduction in any medium, provided the original author(s) and the source are credited. 